International Journal of Pure and Applied Mathematics

Volume 97 No. 3 2014, 311-344

ISSN: 1311-8080 (printed version); ISSN: 1314-3395 (on-line version)

url: http://www.ijpam.eu

doi: http://dx.doi.org/10.12732/ijpam.v97i3.5

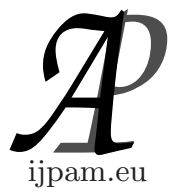

\title{
WAITING TIME IN THE M/M/ $m$ FCFS NONPREEMPTIVE PRIORITY QUEUE WITH IMPATIENT CUSTOMERS
}

\author{
Hideaki Takagi \\ Faculty of Engineering, Information and Systems \\ University of Tsukuba \\ Tsukuba Science City, Ibaraki 305-8573, JAPAN
}

\begin{abstract}
As a possible model for a service center with multiple servers and prioritized impatient customers, we study an $\mathrm{M} / \mathrm{M} / \mathrm{m}$ nonpreemptive priority (first-come, first-served within the same class) queueing system with impatient customers in the steady state. We analyze the waiting time for those customers who are served successfully and the time to abandonment for those customers who leave the system while waiting. Explicit formulas are given for the probabilities of service and abandonment as well as the mean and second moment of the waiting time for customers of each class. The distribution of the waiting time is derived only for customers of the highest priority class. Numerical examples are shown in order to demonstrate the feasibility of computation from theoretical formulas.
\end{abstract}

AMS Subject Classification: 60K25, 68M20, 90B22

Key Words: queue, waiting time, multiserver, nonpreemptive priority, impatient customers, abandonment

\section{Introduction}

We consider a queueing system with $m$ servers and an infinite capacity of the waiting room with $P$ priority classes of customers. Customers of class $p$ arrive

Received: June 28, 2014

(c) 2014 Academic Publications, Ltd. url: www.acadpubl.eu 


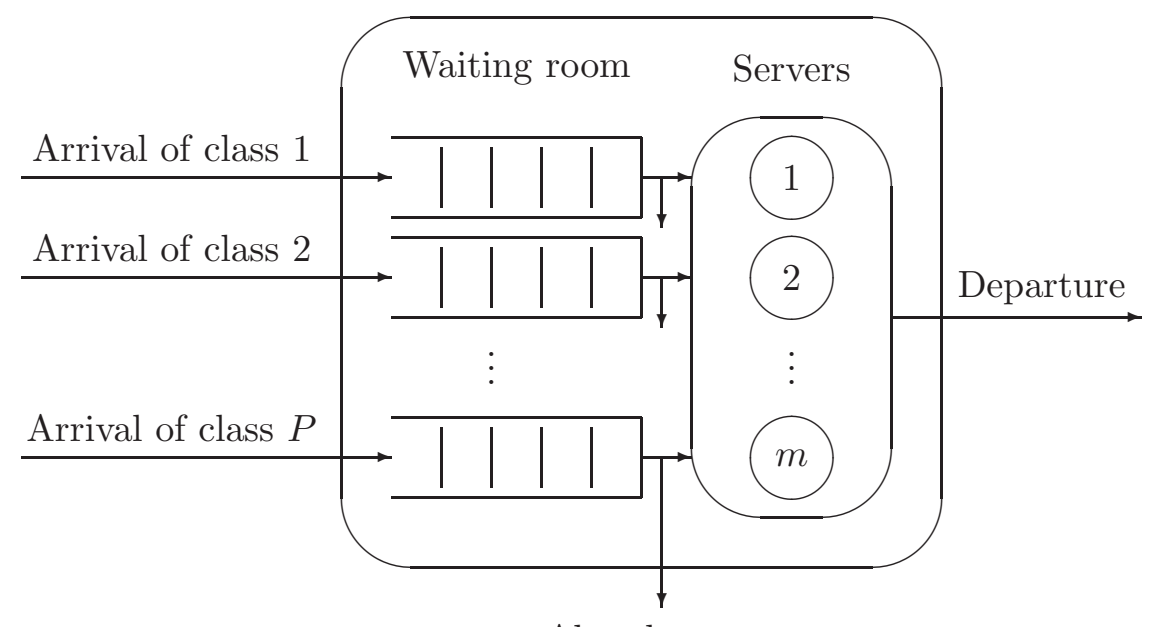

Abandonment

Figure 1: $\mathrm{M} / \mathrm{M} / m$ nonpreemptive priority queue with impatient customers.

in a Poisson process with rate $\lambda_{p}(>0)$ independently of customers of all other classes. Every customer requests a service which has the exponential distribution with mean $1 / \mu$ irrespective of his class. Classes are indexed $1,2, \ldots, P$ such that customers of class $p$ have nonpreemptive priority for the entrance to service over customers of class $q$ if $p<q$. Customers of the same class are served on the first-come, first-served (FCFS) basis. In addition, we assume that each customer in the waiting room leaves the queue (abandons waiting) with probability $\theta \Delta t$ during a short interval $(t, t+\Delta t)$. In other words, the patience time of each customer is exponentially distributed with mean $1 / \theta$ irrespective of his class. Once the service is started, he does not leave the system and his service is not disrupted by the subsequent arrivals of any other customers. Thus we call our system an "M/M/m FCFS nonpreemptive priority queue with impatient customers." The purpose of this paper is to present a stochastic analysis for the waiting time of an arbitrary customer of each class depending on whether he is served or abandons waiting in the steady state of the system. This system is depicted schematically in Figure 1.

The study of waiting times of customers in the basic $\mathrm{M} / \mathrm{M} / \mathrm{m}$ nonpreemptive priority queue (without abandonment) dates back to old days. Cobham [2] derived the mean waiting time for each a customer of each class. Davis [3] obtained the distribution of the waiting time in the form of Laplace transform. More recently, Kella and Yechiali [6] showed an alternative derivation of the 
Laplace transform of the waiting time. They also derived the second moment of the waiting time.

The $\mathrm{M} / \mathrm{M} / m$ queue with impatient customers (without priorities) is studied even in earlier days. Palm [10,11] (cited in [8] and [12]) derived the probability of abandonment and the mean waiting time for an arbitrary customer. (It is mentioned in [9, p. 169] that Engset's main model in his report from 1915 under certain conditions is identical to Palm's model.) The distribution of the waiting time is shown in such books as Gnedenko and Kovalenko [4, p. 64] and Riordan [12, p. 109]. Lately, this system has been used widely as a basic model for the performance evaluation of a call center $[8,15]$. The author [13] recently presented a detailed analysis of the waiting time in the $\mathrm{M} / \mathrm{M} / m /(m+c)$ queue (with a finite waiting room) with impatient customers.

Multi-server priority queues with impatient customers are studied by Brandt and Brandt [1] and by Iravani and Balciog̃lu [5]. They consider the case of two priority classes as a model of a call center. The queueing system studied in the present paper can have an arbitrary number of servers and an arbitrary number of priority classes as the combination of the above-mentioned traditional models. Our system may be used to model a service center with many servers and prioritized customers such as a call center with prioritized customers, a hospital with patients of various degrees of emergency, and so on. The characteristics of the waiting time are among the key performance indices in these service organizations.

We use the following notation for the analysis in this paper.

$$
\begin{gathered}
\lambda_{p}^{+}:=\sum_{k=1}^{p} \lambda_{k} \quad 1 \leq p \leq P \quad ; \quad \lambda:=\lambda_{P}^{+}=\sum_{k=1}^{P} \lambda_{k}, \\
\rho_{p}:=\frac{\lambda_{p}}{m \mu} \quad ; \quad \rho_{p}^{+}:=\sum_{k=1}^{p} \rho_{k}=\frac{\lambda_{p}^{+}}{m \mu} \quad 1 \leq p \leq P, \\
\rho:=\rho_{P}^{+}=\sum_{k=1}^{P} \rho_{k}=\frac{\lambda}{m \mu} \quad ; \quad \tau:=\frac{\theta}{\mu} .
\end{gathered}
$$

In the numerical examples throughout this paper, we assume that

$$
m=5 \quad ; \quad \mu=1 \quad ; \quad \theta=2 \quad ; \quad P=4 \quad ; \quad \lambda_{p}=\frac{\lambda}{P} \quad(1 \leq p \leq P) .
$$

We will show several performance measures against $\lambda$ for the range $0 \leq \lambda \leq 10$ in figures and the range $0 \leq \lambda \leq 50$ or 100 in tables. 


\section{Number of customers in the waiting room}

Let us focus on customers of class $p$. Following an approach in [3], we denote by $Q_{k}(t)$ the probability that all $m$ servers are busy (some of them may be serving customers of classes $p+1, \ldots, P$ ) and there are $k$ customers of classes $1,2, \ldots, p$ in the waiting room at time $t$. For the sake of simplicity in notation, we omit the explicit subscript $p$ for $Q_{k}(t)$ and other symbols below. In addition, we denote by $P_{k}(t)$ the probability that there are $k$ customers of any class in the whole $\mathrm{M} / \mathrm{M} / m$ system at time $t$.

Then, by considering the possible events to occur during a short time interval $(t, t+\Delta t)$, we get the following set of relations:

$$
\begin{aligned}
Q_{0}(t+\Delta t) & =Q_{1}(t)(m \mu+\theta) \Delta t+P_{m-1}(t) \lambda \Delta t \\
& +\left[Q_{0}(t)-P_{m}(t)\right] m \mu \Delta t \\
& +Q_{0}(t)\left[1-\left(m \mu+\lambda_{p}^{+}\right) \Delta t\right]+o(\Delta t), \\
Q_{k}(t+\Delta t) & =Q_{k-1}(t) \lambda_{p}^{+} \Delta t+Q_{k+1}(t)[m \mu+(k+1) \theta] \Delta t \\
& +Q_{k}(t)\left[1-\left(m \mu+k \theta+\lambda_{p}^{+}\right) \Delta t\right]+o(\Delta t) \quad k \geq 1,
\end{aligned}
$$

where $Q_{0}(t)-P_{m}(t)$ is the probability that all servers are busy and there are no customers of classes $1,2, \ldots, p$ but at least one customer of classes $p+1, \ldots, P$ in the waiting room at time $t$. We also have the following set of relations:

$$
\begin{aligned}
P_{0}(t+\Delta t) & =P_{0}(t)(1-\lambda \Delta t)+P_{1}(t) \mu \Delta t+o(\Delta t), \\
P_{k}(t+\Delta t) & =P_{k-1}(t) \lambda \Delta t+P_{k}(t)[1-(\lambda+k \mu) \Delta t] \\
& +P_{k+1}(t)(k+1) \mu \Delta t+o(\Delta t) \quad 1 \leq k \leq m-1, \\
P_{k}(t+\Delta t) & =P_{k-1}(t) \lambda \Delta t+P_{k}(t)\{1-[\lambda+m \mu+(k-m) \theta] \Delta t\} \\
& +P_{k+1}(t)[m \mu+(k+1-m) \theta] \Delta t+o(\Delta t) \quad k \geq m .
\end{aligned}
$$

By letting $\Delta t \rightarrow 0$, we obtain the following set of difference-differential equations for $\left\{Q_{k}(t)\right\}$ :

$$
\begin{aligned}
\frac{d Q_{0}(t)}{d t} & =(m \mu+\theta) Q_{1}(t)-\lambda_{p}^{+} Q_{0}(t)+\lambda P_{m-1}(t)-m \mu P_{m}(t), \\
\frac{d Q_{k}(t)}{d t} & =\lambda_{p}^{+} Q_{k-1}(t)+[m \mu+(k+1) \theta] Q_{k+1}(t) \\
& -\left(m \mu+k \theta+\lambda_{p}^{+}\right) Q_{k}(t) \quad k \geq 1
\end{aligned}
$$


and the following set of difference-differential equations for $\left\{P_{k}(t)\right\}$ :

$$
\begin{aligned}
\frac{d P_{0}(t)}{d t} & =-\lambda P_{0}(t)+\mu P_{1}(t), \\
\frac{d P_{k}(t)}{d t} & =\lambda P_{k-1}(t)-(\lambda+k \mu) P_{k}(t)+(k+1) \mu P_{k+1}(t) \\
\frac{d P_{k}(t)}{d t} & =\lambda P_{k-1}(t)-[\lambda+m \mu+(k-m) \theta] P_{k}(t) \\
& +[m \mu+(k+1-m) \theta] P_{k+1}(t) \quad k \geq m .
\end{aligned}
$$

We now consider the steady-state probabilities defined by

$$
Q_{k}=\lim _{t \rightarrow \infty} Q_{k}(t) \quad ; \quad P_{k}=\lim _{t \rightarrow \infty} P_{k}(t) \quad k \geq 0 .
$$

Then we have

$$
\begin{aligned}
m \mu P_{m}+\lambda_{p}^{+} Q_{0} & =\lambda P_{m-1}+(m \mu+\theta) Q_{1} \\
\left(m \mu+k \theta+\lambda_{p}^{+}\right) Q_{k} & =\lambda_{p}^{+} Q_{k-1}+[m \mu+(k+1) \theta] Q_{k+1} \quad k \geq 1,
\end{aligned}
$$

and

$$
\begin{aligned}
\lambda P_{0} & =\mu P_{1} \\
(\lambda+k \mu) P_{k} & =\lambda P_{k-1}+(k+1) \mu P_{k+1} \quad 1 \leq k \leq m-1, \\
{[\lambda+m \mu+(k-m) \theta] P_{k} } & =\lambda P_{k-1}+[m \mu+(k+1-m) \theta] P_{k+1} \\
&
\end{aligned}
$$

The latter set of equations reduces to the simplified set:

$$
\begin{aligned}
\lambda P_{k-1} & =k \mu P_{k} \quad 1 \leq k \leq m, \\
\lambda P_{k-1} & =[m \mu+(k-m) \theta] P_{k} \quad k \geq m+1,
\end{aligned}
$$

which has the solution

$$
P_{k}= \begin{cases}P_{0} \frac{(m \rho)^{k}}{k !} & 1 \leq k \leq m, \\ P_{m} \frac{\rho^{k-m}}{\prod_{j=0}^{k-m}(1+j \tau / m)} & k \geq m+1,\end{cases}
$$


where, from the normalization condition $\sum_{k=0}^{\infty} P_{k}=1$, we have

$$
\frac{1}{P_{0}}=\sum_{k=0}^{m-1} \frac{(m \rho)^{k}}{k !}+\frac{(m \rho)^{m}}{m !} \sum_{k=0}^{\infty} \frac{\rho^{k}}{\prod_{j=0}^{k}(1+j \tau / m)} .
$$

We note that $P_{0}$ is the probability that the whole system is empty at an arbitrary time.

Now that we have the relation $\lambda P_{m-1}=m \mu P_{m}$, the former set of equations for $\left\{Q_{k}\right\}$ reduces to the following:

$$
\lambda_{p}^{+} Q_{k-1}=(m \mu+k \theta) Q_{k} \quad k \geq 1 .
$$

Thus we get

$$
Q_{k}=Q_{0} \frac{\left(\rho_{p}^{+}\right)^{k}}{\prod_{j=1}^{k}(1+j \tau / m)} \quad k \geq 1 .
$$

The probability $Q_{0}$ that all servers are busy and there are no customers of classes $1,2, \ldots, p$ in the waiting room at an arbitrary time is determined from the condition

$$
P\{W>0\}=\sum_{k=0}^{\infty} Q_{k}
$$

where $W$ denotes the waiting time of an arbitrary customer so that, due to the PASTA (Poisson arrivals see time averages) property, $P\{W>0\}$ is the probability that all servers are busy at an arbitrary time. It is then given by

$$
P\{W>0\}=\sum_{k=m}^{\infty} P_{k}=P_{0} \frac{(m \rho)^{m}}{m !} \sum_{k=0}^{\infty} \frac{\rho^{k}}{\prod_{j=0}^{k}(1+j \tau / m)} .
$$

Eq. (3) is an extension of Erlang's C formula to the queue with impatient customers. Thus we can get $Q_{0}$ to be used in Eq. (2) as follows:

$$
Q_{0}=P\{W>0\} / \sum_{k=0}^{\infty} \frac{\left(\rho_{p}^{+}\right)^{k}}{\prod_{j=0}^{k}(1+j \tau / m)} .
$$

Note that $P_{0}$ and $P\{W>0\}$ do not depend on the class $p$ while $Q_{0}$ does depend on $p$.

All the summations to infinity appearing in these expressions converge as

$$
\sum_{k=0}^{\infty} \frac{\rho^{k}}{\prod_{j=0}^{k}(1+j \tau / m)}<1+\sum_{k=1}^{\infty} \frac{\rho^{k}}{\prod_{j=1}^{k}(j \tau / m)}=\sum_{k=0}^{\infty} \frac{(m \rho / \tau)^{k}}{k !}=e^{m \rho / \tau} .
$$


Therefore, our system with impatient customers $(\theta>0)$ is always stable, because the more customers wait the longer they wait and the more they abandon waiting. In fact, the following sums can be expressed in terms of the incomplete gamma functions [8]:

$$
\begin{aligned}
\sum_{k=0}^{\infty} \frac{\rho^{k}}{\prod_{j=0}^{k}(1+j \tau / m)} & =A\left(\frac{m}{\tau}, \frac{m \rho}{\tau}\right), \\
\frac{\tau}{m \rho} \sum_{k=1}^{\infty} \frac{k \rho^{k}}{\prod_{j=1}^{k}(1+j \tau / m)} & =\left(1-\frac{1}{\rho}\right) A\left(\frac{m}{\tau}, \frac{m \rho}{\tau}\right)+\frac{1}{\rho},
\end{aligned}
$$

where

$$
A(x, y):=\frac{x e^{y}}{y^{x}} \gamma(x, y) \quad ; \quad \gamma(x, y):=\int_{0}^{y} t^{x-1} e^{-t} d t \quad x, y>0 .
$$

The mean number of customers of classes $1,2, \ldots, p$ present in the waiting room is given by

$$
E\left[L_{p}^{+}\right]=\sum_{k=1}^{\infty} k Q_{k}=Q_{0} \sum_{k=1}^{\infty} \frac{k\left(\rho_{p}^{+}\right)^{k}}{\prod_{j=1}^{k}(1+j \tau / m)} \quad 1 \leq p \leq P .
$$

The mean number of customers of class $p$ present in the waiting room is then given by

$$
E\left[L^{(1)}\right]=E\left[L_{1}^{+}\right] \quad ; \quad E\left[L^{(p)}\right]=E\left[L_{p}^{+}\right]-E\left[L_{p-1}^{+}\right] \quad 2 \leq p \leq P .
$$

Figure 2 plots $E\left[L^{(p)}\right]$ for class $p=1$ through 4 against the arrival rate $\lambda$ for each class in the numerical example defined in Section 1.

The number of customers of all classes being served at an arbitrary time, denoted by $S$, has the probability distribution

$$
P\{S=k\}= \begin{cases}P_{0} \frac{(\lambda / \mu)^{k}}{k !} & 0 \leq k \leq m-1, \\ P\{W>0\} & k=m .\end{cases}
$$

Therefore, the mean number of customers of all classes being served at an arbitrary time is given by

$$
E[S]=\sum_{k=1}^{m} k P\{S=k\}=P_{0} \sum_{k=1}^{m-1} \frac{(\lambda / \mu)^{k}}{(k-1) !}+m P\{W>0\} .
$$

Figure 3 plots $E[S]$ against the arrival rate $\lambda$ in the numerical example defined in Section 1. 


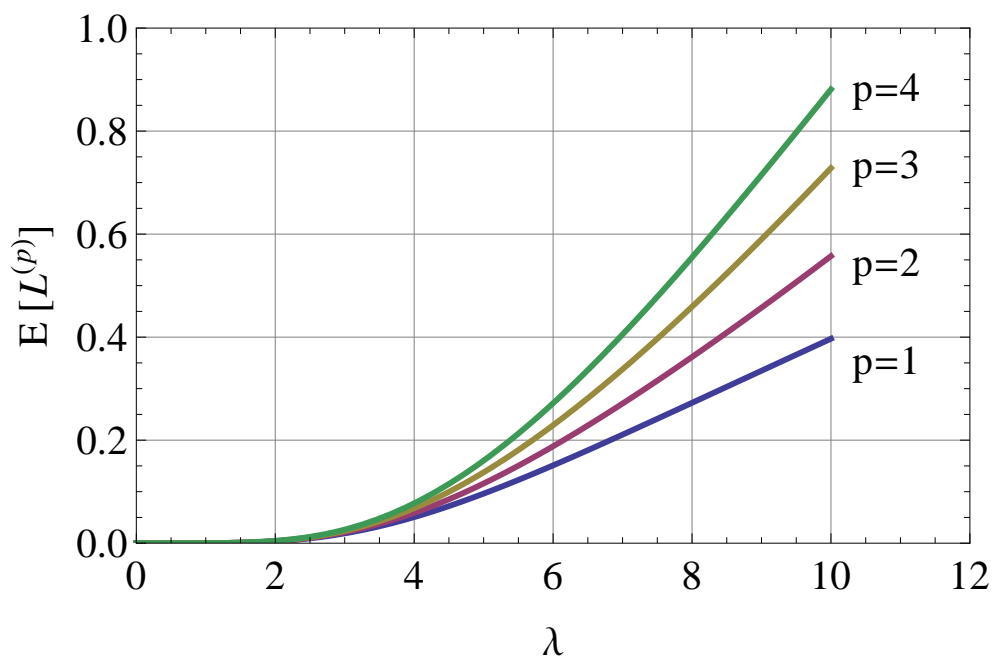

Figure 2: Mean number of customers of class $p$ in the waiting room.

\section{First passage time to service or abandonment}

We now focus on a tagged customer of class $p$ in state $k$, which means that there are $k$ other customers of classes $1,2, \ldots, p$ in the waiting room who are to be served before the tagged customer. The state transition diagram for the tagged customer is shown in Figure 4. We consider the first passage time in this one-dimensional birth-and-death process with two absorbing states, namely the state "service" denoted by "Sr" and the state "abandonment" denoted by "Ab." This approach is an extension of the one taken by Whitt [15]. It was also adopted by the author [13]. See, for example, Kulkarni [7, Chapter 4] and Taylor and Karlin [14, Chapter III] for the treatment of discrete-time Markov chains with absorbing states.

For class $p(\geq 2)$, we define the state transition probabilities

$$
\begin{aligned}
& \alpha_{k}=\frac{m \mu+k \theta}{m \mu+(k+1) \theta+\lambda_{p-1}^{+}} \quad ; \quad \beta_{k}=\frac{\theta}{m \mu+(k+1) \theta+\lambda_{p-1}^{+}}, \\
& 1-\alpha_{k}-\beta_{k}=\frac{\lambda_{p-1}^{+}}{m \mu+(k+1) \theta+\lambda_{p-1}^{+}} \quad k \geq 0 .
\end{aligned}
$$

The Laplace transform of the probability density function (pdf) for the time 


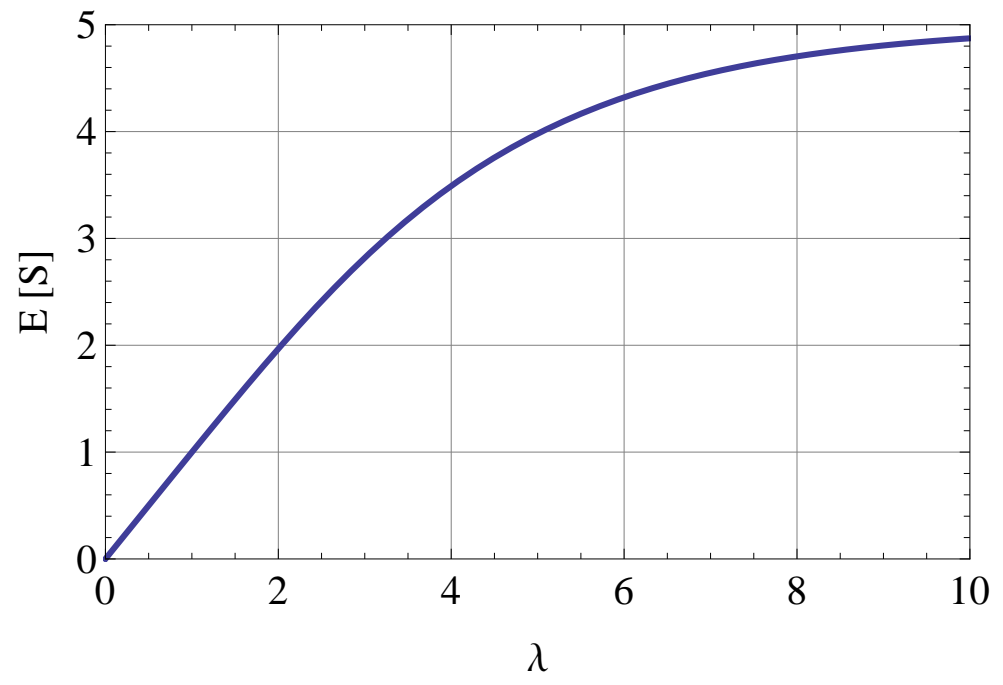

Figure 3: Mean number of customers in service.

that the tagged customer stays in state $k$ is given by

$$
B_{k}^{*}(s)=\frac{m \mu+(k+1) \theta+\lambda_{p-1}^{+}}{s+m \mu+(k+1) \theta+\lambda_{p-1}^{+}} \quad k \geq 0
$$

with its first and second moments:

$$
\begin{gathered}
b_{k}=\frac{1}{m \mu+(k+1) \theta+\lambda_{p-1}^{+}} \quad ; \quad b_{k}^{(2)}=\frac{2}{\left[m \mu+(k+1) \theta+\lambda_{p-1}^{+}\right]^{2}} \\
k \geq 1 .
\end{gathered}
$$

We denote by $W_{k}^{*}(s, \mathrm{Sr})$ the joint probability of successful service and the Laplace transform of the pdf of the waiting time for a customer of class $p$ who has $k$ other customers of classes $1,2, \ldots, p$ before him in the waiting room. Applying the first step analysis [7, p. 162], [14, p. 116] to state $k$, we have the following set of equations for $\left\{W_{k}^{*}(s, \mathrm{Sr}) ; k \geq 0\right\}$ :

$$
\begin{gathered}
W_{0}^{*}(s, \mathrm{Sr})=B_{0}^{*}(s)\left[\alpha_{0}+\left(1-\alpha_{0}-\beta_{0}\right) W_{1}^{*}(s, \mathrm{Sr})\right] \\
W_{k}^{*}(s, \mathrm{Sr})=B_{k}^{*}(s)\left[\alpha_{k} W_{k-1}^{*}(s, \mathrm{Sr})+\left(1-\alpha_{k}-\beta_{k}\right) W_{k+1}^{*}(s, \mathrm{Sr})\right] \\
\quad k \geq 1 .
\end{gathered}
$$




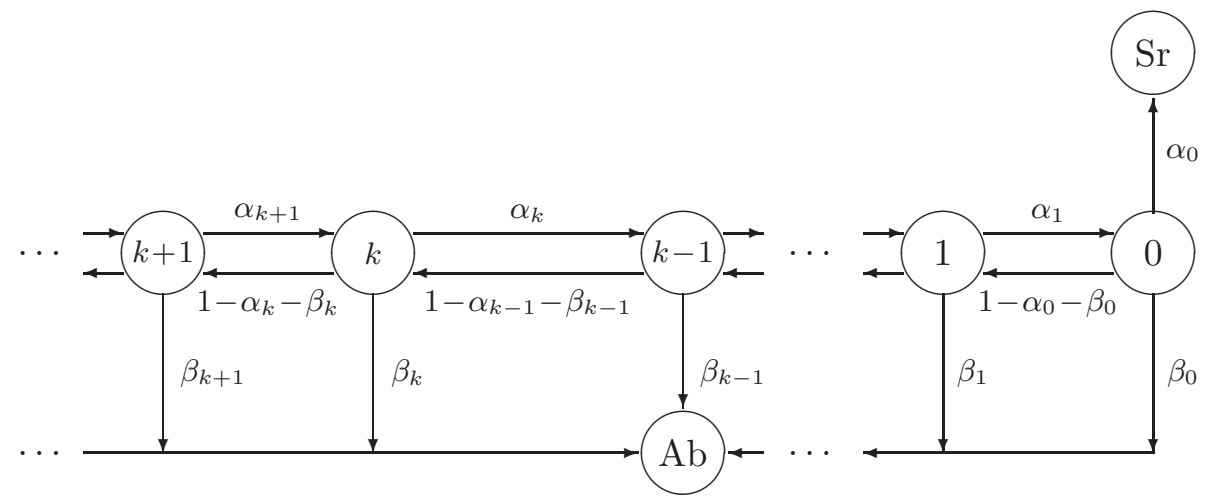

Figure 4: State transition diagram for a customer until either service or abandonment.

Similarly, let $W_{k}^{*}(s, \mathrm{Ab})$ be the joint probability of abandonment and the Laplace transform of the pdf of the time to the abandonment for a customer of class $p$ who has $k$ other customers of classes $1,2, \ldots, p$ before him in the waiting room. Then we have the following set of equations for $\left\{W_{k}^{*}(s, \mathrm{Ab}) ; k \geq 0\right\}$ :

$$
\begin{aligned}
W_{0}^{*}(s, \mathrm{Ab}) & =B_{0}^{*}(s)\left[\beta_{0}+\left(1-\alpha_{0}-\beta_{0}\right) W_{1}^{*}(s, \mathrm{Ab})\right], \\
W_{k}^{*}(s, \mathrm{Ab}) & =B_{k}^{*}(s)\left[\alpha_{k} W_{k-1}^{*}(s, \mathrm{Ab})+\beta_{k}\right. \\
& \left.+\left(1-\alpha_{k}-\beta_{k}\right) W_{k+1}^{*}(s, \mathrm{Ab})\right] \quad k \geq 1 .
\end{aligned}
$$

If we denote by $W_{k}^{*}(s)$ the Laplace transform of the pdf of the unconditional waiting time for a customer of class $p$ who has $k$ other customers of classes $1,2, \ldots, p$ before him in the waiting room whether he gets served or abandons waiting, we have

$$
W_{k}^{*}(s)=W_{k}^{*}(s, \mathrm{Sr})+W_{k}^{*}(s, \mathrm{Ab}) \quad k \geq 0 .
$$

Adding Eq. (7) and Eq. (8) term by term, we get the following set of equations for $\left\{W_{k}^{*}(s) ; k \geq 0\right\}$ :

$$
\begin{aligned}
W_{0}^{*}(s) & =B_{0}^{*}(s)\left[\alpha_{0}+\beta_{0}+\left(1-\alpha_{0}-\beta_{0}\right) W_{1}^{*}(s)\right], \\
W_{k}^{*}(s)=B_{k}^{*}(s)\left[\alpha_{k} W_{k-1}^{*}(s)+\beta_{k}+\left(1-\alpha_{k}-\beta_{k}\right) W_{k+1}^{*}(s)\right] & k \geq 1 .
\end{aligned}
$$


Therefore we can show that the following relation holds:

$$
W_{k}^{*}(s, \mathrm{Ab})=(\theta / s)\left[1-W_{k}^{*}(s)\right] \quad k \geq 0 .
$$

The highest priority class $p=1$ needs special treatment as if $\lambda_{0}^{+}=0$ in the above. Then we have

$$
\alpha_{k}=\frac{m \mu+k \theta}{m \mu+(k+1) \theta} \quad ; \quad \beta_{k}=\frac{\theta}{m \mu+(k+1) \theta} \quad ; \quad 1-\alpha_{k}-\beta_{k}=0
$$

and

$$
B_{k}^{*}(s)=\frac{m \mu+(k+1) \theta}{s+m \mu+(k+1) \theta}
$$

with

$$
b_{k}=\frac{1}{m \mu+(k+1) \theta} \quad ; \quad b_{k}^{(2)}=\frac{2}{[m \mu+(k+1) \theta]^{2}} .
$$

In this case we have

$$
\begin{array}{r}
W_{0}^{*}(s, \mathrm{Sr})=\alpha_{0} B_{0}^{*}(s), \quad W_{k}^{*}(s, \mathrm{Sr})=\alpha_{k} B_{k}^{*}(s) W_{k-1}^{*}(s, \mathrm{Sr}) \\
k \geq 1
\end{array}
$$

and

$$
\begin{gathered}
W_{0}^{*}(s, \mathrm{Ab})=\beta_{0} B_{0}^{*}(s), \quad W_{k}^{*}(s, \mathrm{Ab})=B_{k}^{*}(s)\left[\alpha_{k} W_{k-1}^{*}(s, \mathrm{Ab})+\beta_{k}\right] \\
k \geq 1
\end{gathered}
$$

We will show explicit expressions for $W_{k}^{*}(s, \mathrm{Sr})$ and $W_{k}^{*}(s, \mathrm{Ab})$ as the solution to Eqs. (11) and (12) as well as their inverse transforms in Section 7. We can show that the relation in Eq. (10) also holds in this case.

Now, let us define

$$
\begin{aligned}
W^{*}(s, \mathrm{Ab}) & :=\sum_{k=0}^{\infty} Q_{k} W_{k}^{*}(s, \mathrm{Ab}), \\
W^{*}(s) & :=P\{W=0\}+\sum_{k=0}^{\infty} Q_{k} W_{k}^{*}(s) .
\end{aligned}
$$

Then, from Eq. (10), we get the relation

$$
W^{*}(s, \mathrm{Ab})=(\theta / s)\left[1-W^{*}(s)\right]
$$


for customers of each class. If we introduce the pdf's $f_{W}(t, \mathrm{Ab})$ and $f_{W}(t)$ by

$$
\begin{aligned}
W^{*}(s, \mathrm{Ab}) & =\int_{0}^{\infty} e^{-s t} f_{W}(t, \mathrm{Ab}) d t \\
W^{*}(s) & =P\{W=0\}+\int_{0}^{\infty} e^{-s t} f_{W}(t) d t,
\end{aligned}
$$

we can show that Eq. (13) is equivalent to the relation

$$
f_{W}(t, \mathrm{Ab})=\theta P\{W>t\} \quad t \geq 0 .
$$

This makes sense because a customer who has waited $t$ time units abandons waiting with probability $\theta \Delta t$ in the time interval $(t, t+\Delta t)$.

\section{Probabilities of service and abandonment}

We first study the probability that an arbitrary customer gets served and the probability that an arbitrary customer abandons waiting without being served.

\subsection{Probability of service}

We denote by $P_{k}\{\mathrm{Sr}\}$ the probability that a customer of class $p$ who has $k$ other customers of classes $1,2, \ldots, p$ before him in the waiting room is eventually served:

$$
P_{k}\{\mathrm{Sr}\}=W_{k}^{*}(0, \mathrm{Sr}) \quad k \geq 0 .
$$

For customers of class $p(\geq 2)$, from Eq. (7) we get the following set of equations for $\left\{P_{k}\{\mathrm{Sr}\} ; k \geq 0\right\}$ :

$$
\begin{aligned}
& P_{0}\{\mathrm{Sr}\}=\alpha_{0}+\left(1-\alpha_{0}-\beta_{0}\right) P_{1}\{\mathrm{Sr}\} \\
& P_{k}\{\mathrm{Sr}\}=\alpha_{k} P_{k-1}\{\mathrm{Sr}\}+\left(1-\alpha_{k}-\beta_{k}\right) P_{k+1}\{\mathrm{Sr}\} \quad k \geq 1,
\end{aligned}
$$

which can be written as

$$
\begin{aligned}
P_{0}\{\mathrm{Sr}\}-\xi_{1} P_{1}\{\mathrm{Sr}\} & =\eta_{0}\left(1-\xi_{0} P_{0}\{\mathrm{Sr}\}\right) \\
P_{k}\{\mathrm{Sr}\}-\xi_{k+1} P_{k+1}\{\mathrm{Sr}\} & =\eta_{k}\left(P_{k-1}\{\mathrm{Sr}\}-\xi_{k} P_{k}\{\mathrm{Sr}\}\right) \quad k \geq 1
\end{aligned}
$$

by introducing the notation

$$
\xi_{k}:=\frac{\lambda_{p-1}^{+}}{m \mu+k \theta} \quad ; \quad \eta_{k}:=\frac{m \mu+k \theta}{m \mu+(k+1) \theta} \quad k \geq 0 .
$$


Thus we have

$$
\begin{aligned}
P_{k}\{\mathrm{Sr}\}-\xi_{k+1} P_{k+1}\{\mathrm{Sr}\} & =\eta_{k} \eta_{k-1} \cdots \eta_{1} \eta_{0}\left(1-\xi_{0} P_{0}\{\mathrm{Sr}\}\right) \\
& =\frac{m \mu}{m \mu+(k+1) \theta}\left(1-\frac{\lambda_{p-1}^{+}}{m \mu} P_{0}\{\mathrm{Sr}\}\right)
\end{aligned}
$$

from which we get by recursion

$$
\begin{aligned}
P_{k}\{\mathrm{Sr}\} & =\frac{P_{k-1}\{\mathrm{Sr}\}}{\xi_{k}}+P_{0}\{\mathrm{Sr}\}-\frac{1}{\xi_{0}} \\
& =\frac{P_{k-2}\{\mathrm{Sr}\}}{\xi_{k} \xi_{k-1}}+\left(P_{0}\{\mathrm{Sr}\}-\frac{1}{\xi_{0}}\right)\left(\frac{1}{\xi_{k}}+1\right) \\
& =\cdots \\
& =\left[P_{0}\{\mathrm{Sr}\}+\left(P_{0}\{\mathrm{Sr}\}-\frac{1}{\xi_{0}}\right) \sum_{j=1}^{k} \prod_{l=1}^{j} \xi_{l} / \prod_{j=1}^{k} \xi_{j}\right.
\end{aligned}
$$

for $k \geq 1$. In order to determine $P_{0}\{\mathrm{Sr}\}$, we conjecture that

$$
\lim _{k \rightarrow \infty} P_{k}\{\mathrm{Sr}\}=0
$$

which means that the probability that a customer with so many others before him will be served is zero. Thus we determine

$$
P_{0}\{\mathrm{Sr}\}=\sum_{j=1}^{\infty} \prod_{l=1}^{j} \xi_{l} / \sum_{j=0}^{\infty} \prod_{l=0}^{j} \xi_{l} .
$$

We then get

$$
\begin{aligned}
& P_{k}\{\mathrm{Sr}\}=\sum_{j=k+1}^{\infty} \prod_{l=k+1}^{j} \xi_{l} / \sum_{j=0}^{\infty} \prod_{l=0}^{j} \xi_{l} \\
&=\sum_{j=k+1}^{\infty} \frac{\left(\rho_{p-1}^{+}\right)^{j-k}}{\prod_{l=k+1}^{j}(1+l \tau / m)} / \sum_{j=0}^{\infty} \frac{\left(\rho_{p-1}^{+}\right)^{j+1}}{\prod_{l=0}^{j}(1+l \tau / m)} \\
& k \geq 0 .
\end{aligned}
$$

For customers of the highest priority class $p=1$, we have

$$
P_{0}\{\mathrm{Sr}\}=\alpha_{0}, \quad P_{k}\{\mathrm{Sr}\}=\alpha_{k} P_{k-1}\{\mathrm{Sr}\} \quad k \geq 1,
$$


which simply yields

$$
P_{k}\{\mathrm{Sr}\}=\frac{m \mu}{m \mu+(k+1) \theta} \quad k \geq 0 .
$$

The probability that a customer of class $p$ gets served is given by

$$
P\left\{\mathrm{Sr}^{(p)}\right\}=P\{W=0\}+\sum_{k=0}^{\infty} Q_{k} P_{k}\{\mathrm{Sr}\},
$$

where $P_{k}\{\mathrm{Sr}\}$ in Eq. (16) is used for class $2 \leq p \leq P$ and $P_{k}\{\mathrm{Sr}\}$ in Eq. (17) is used for class $p=1$.

We note the relation

$$
\sum_{p=1}^{P} \lambda_{p} P\left\{\mathrm{Sr}^{(p)}\right\}=\mu E[S],
$$

where $E[S]$ is the mean number of customers of all classes being served at an arbitrary time given in Eq. (6). Each side of Eq. (19) shows the throughput of the system, that is the mean number of customers successfully served per unit time.

Figure 5 plots $P\left\{\mathrm{Sr}^{(p)}\right\}$ for class $p=1$ through 4 against the arrival rate $\lambda$ in the numerical example defined in Section 1.

\subsection{Probability of abandonment}

We denote by $P_{k}\{\mathrm{Ab}\}$ the probability that a customer of class $p$ who has $k$ other customers of classes $1,2, \ldots, p$ before him in the waiting room abandons waiting:

$$
P_{k}\{\mathrm{Ab}\}=W_{k}^{*}(0, \mathrm{Ab}) \quad k \geq 0 .
$$

For customers of class $p(\geq 2)$, from Eq. (8) we get the following set of equations for $\left\{P_{k}\{\mathrm{Ab}\} ; k \geq 0\right\}$ :

$$
\begin{aligned}
& P_{0}\{\mathrm{Ab}\}=\beta_{0}+\left(1-\alpha_{0}-\beta_{0}\right) P_{1}\{\mathrm{Ab}\} \\
& P_{k}\{\mathrm{Ab}\}=\alpha_{k} P_{k-1}\{\mathrm{Ab}\}+\beta_{k}+\left(1-\alpha_{k}-\beta_{k}\right) P_{k+1}\{\mathrm{Ab}\} \quad k \geq 1 .
\end{aligned}
$$

It is clear that this set of equations is satisfied by

$$
P_{k}\{\mathrm{Ab}\}=1-P_{k}\{\mathrm{Sr}\} \quad k \geq 0,
$$

where $P_{k}\{\mathrm{Sr}\}$ is given in Eq. (16). 


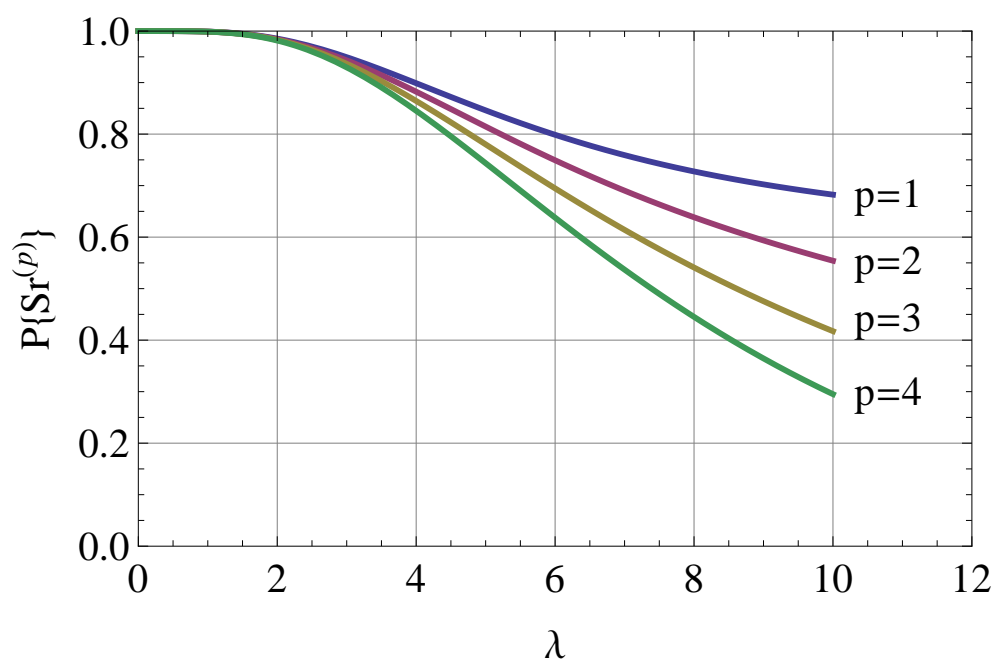

Figure 5: Probability of service for customers of class $p$.

For customers of the highest priority class $p=1$, we have

$$
P_{0}\{\mathrm{Ab}\}=\beta_{0}, \quad P_{k}\{\mathrm{Ab}\}=\alpha_{k} P_{k-1}\{\mathrm{Ab}\}+\beta_{k} \quad k \geq 1,
$$

which are satisfied by

$$
P_{k}\{\mathrm{Ab}\}=\frac{(k+1) \theta}{m \mu+(k+1) \theta}=1-P_{k}\{\mathrm{Sr}\} \quad k \geq 0,
$$

where $P_{k}\{\mathrm{Sr}\}$ is given in Eq. (17).

The probability that a customer of class $p$ abandons waiting is given by

$$
P\left\{\mathrm{Ab}^{(p)}\right\}=\sum_{k=0}^{\infty} Q_{k} P_{k}\{\mathrm{Ab}\}=1-P\left\{\mathrm{Sr}^{(p)}\right\},
$$

where $P_{k}\{\mathrm{Ab}\}$ in Eq. (20) is used for class $2 \leq p \leq P$ and $P_{k}\{\mathrm{Ab}\}$ in Eq. (21) is used for class $p=1$. Numerical values for $P\left\{\mathrm{Ab}^{(p)}\right\}$ can be seen from Figure 6 for $P\left\{\mathrm{Sr}^{(p)}\right\}$.

We can confirm the following relation numerically:

$$
\lambda_{p} P\left\{\mathrm{Ab}^{(p)}\right\}=\theta E\left[L^{(p)}\right] \quad 1 \leq p \leq P,
$$

where $E\left[L^{(p)}\right]$ is the mean number of customers of class $p$ present in the waiting room given in (5). Therefore, each side of Eq. (23) shows the mean number of customers of class $p$ who abandon waiting per unit time. 
Substituting Eqs. (22) and (23) into Eq. (19), we get the relation

$$
\lambda=\theta \sum_{p=1}^{P} E\left[L^{(p)}\right]+\mu E[S],
$$

which shows the balance of input and output rates for all customers.

Figure 6 plots $P\left\{\mathrm{Ab}^{(p)}\right\}$ for class $p=1$ through 4 against the arrival rate $\lambda$ for each class in the numerical example defined in Section 1. It is also tabulated in Table 1.

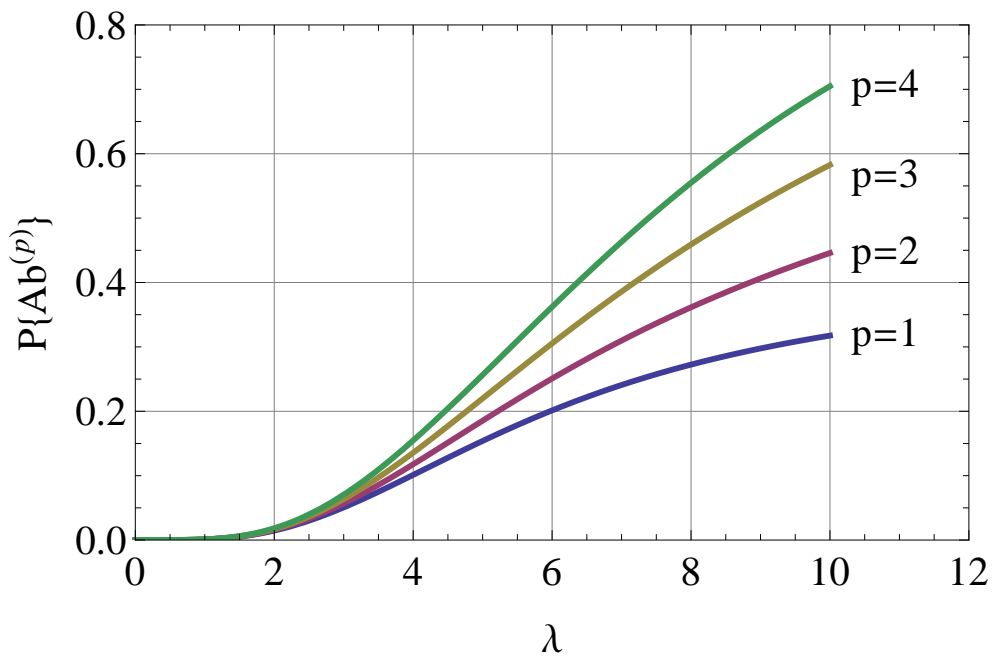

Figure 6: Probability of abandonment for customers of class $p$.

\section{Mean waiting times}

Let us proceed to study the mean time that an arbitrary customer spends in the waiting room. We consider separately the mean waiting time of served customers and the mean time to abandonment for the customers who abandon waiting in the waiting room.

\subsection{Mean waiting time of served customers}

We denote by $E\left[W_{k}\right.$, Sr $]$ the joint probability that a customer of class $p$ who has $k$ other customers of classes $1,2, \ldots, p$ before him in the waiting room is 
Table 1: Probability of abandonment $P\left\{\mathrm{Ab}^{(p)}\right\}$.

\begin{tabular}{rcccc}
\hline$\lambda$ & $P\left\{\mathrm{Ab}^{(1)}\right\}$ & $P\left\{\mathrm{Ab}^{(2)}\right\}$ & $P\left\{\mathrm{Ab}^{(3)}\right\}$ & $P\left\{\mathrm{Ab}^{(4)}\right\}$ \\
\hline 0 & 0.0000 & 0.0000 & 0.0000 & 0.0000 \\
5 & 0.1539 & 0.1854 & 0.2200 & 0.2565 \\
10 & 0.3175 & 0.4458 & 0.5826 & 0.7048 \\
15 & 0.3777 & 0.5935 & 0.7892 & 0.9102 \\
20 & 0.4173 & 0.7038 & 0.9021 & 0.9771 \\
25 & 0.4545 & 0.7921 & 0.9587 & 0.9948 \\
30 & 0.4912 & 0.8594 & 0.9838 & 0.9989 \\
35 & 0.5270 & 0.9078 & 0.9940 & 0.9998 \\
40 & 0.5613 & 0.9409 & 0.9979 & 1.0000 \\
45 & 0.5935 & 0.9628 & 0.9993 & 1.0000 \\
50 & 0.6234 & 0.9769 & 0.9998 & 1.0000 \\
60 & 0.6753 & 0.9914 & 1.0000 & 1.0000 \\
70 & 0.7174 & 0.9969 & 1.0000 & 1.0000 \\
80 & 0.7511 & 0.9989 & 1.0000 & 1.0000 \\
90 & 0.7781 & 0.9996 & 1.0000 & 1.0000 \\
100 & 0.8001 & 0.9999 & 1.0000 & 1.0000 \\
\hline
\end{tabular}

eventually served and his mean waiting time, which is given by

$$
E\left[W_{k}, \mathrm{Sr}\right]=-W_{k}^{*(1)}(0, \mathrm{Sr}) \quad k \geq 0,
$$

where, for a differentiable function $f(x)$, we define

$$
f^{(n)}(0):=\left.\frac{d^{n} f(x)}{d x^{n}}\right|_{x=0} \quad n=1,2, \ldots
$$

For customers of class $p(\geq 2)$, from Eq. (7) we can derive the following set of equations for $\left\{E\left[W_{k}, \mathrm{Sr}\right] ; k \geq 0\right\}$ :

$$
\begin{aligned}
E\left[W_{0}, \mathrm{Sr}\right] & =b_{0} P_{0}\{\mathrm{Sr}\}+\left(1-\alpha_{0}-\beta_{0}\right) E\left[W_{1}, \mathrm{Sr}\right], \\
E\left[W_{k}, \mathrm{Sr}\right]=b_{k} P_{k}\{\mathrm{Sr}\}+\alpha_{k} E\left[W_{k-1}, \mathrm{Sr}\right]+\left(1-\alpha_{k}-\beta_{k}\right) E\left[W_{k+1}, \mathrm{Sr}\right] & k \geq 1,
\end{aligned}
$$

which can be written as

$$
E\left[W_{0}, \mathrm{Sr}\right]-\xi_{1} E\left[W_{1}, \mathrm{Sr}\right]=-\eta_{0} \xi_{0} E\left[W_{0}, \mathrm{Sr}\right]+\frac{P_{0}\{\mathrm{Sr}\}}{m \mu+\theta},
$$




$$
\begin{aligned}
E\left[W_{k}, \mathrm{Sr}\right]-\xi_{k+1} E\left[W_{k+1}, \mathrm{Sr}\right] & =\eta_{k}\left(E\left[W_{k-1}, \mathrm{Sr}\right]-\xi_{k} E\left[W_{k}, \mathrm{Sr}\right]\right) \\
& +\frac{P_{k}\{\mathrm{Sr}\}}{m \mu+(k+1) \theta} \quad k \geq 1
\end{aligned}
$$

with the notation for $\xi_{k}$ and $\eta_{k}$ given in Eq. (15). We then have

$$
\begin{aligned}
E\left[W_{k}, \mathrm{Sr}\right]-\xi_{k+1} E\left[W_{k+1}, \mathrm{Sr}\right] \\
=\eta_{k} \eta_{k-1} \cdots \eta_{0}\left(0-\xi_{0} E\left[W_{0}, \mathrm{Sr}\right]\right)+\sum_{j=1}^{k} \frac{\eta_{j} \cdots \eta_{k}}{m \mu+j \theta} P_{j-1}\{\mathrm{Sr}\} \\
\quad+\frac{P_{k}\{\mathrm{Sr}\}}{m \mu+(k+1) \theta} \\
=\frac{1}{m \mu+(k+1) \theta}\left(\sum_{j=0}^{k} P_{j}\{\mathrm{Sr}\}-\lambda_{p-1}^{+} E\left[W_{0}, \mathrm{Sr}\right]\right),
\end{aligned}
$$

from which we get by recursion

$$
\begin{aligned}
& E\left[W_{k}, \mathrm{Sr}\right] \\
& =\frac{E\left[W_{k-1}, \mathrm{Sr}\right]}{\xi_{k}}+E\left[W_{0}, \mathrm{Sr}\right]-\frac{1}{\lambda_{p-1}^{+}} \sum_{l=0}^{k-1} P_{l}\{\mathrm{Sr}\} \\
& =\frac{E\left[W_{k-2}, \mathrm{Sr}\right]}{\xi_{k} \xi_{k-1}}+E\left[W_{0}, \mathrm{Sr}\right]\left(\frac{1}{\xi_{k}}+1\right)-\frac{1}{\lambda_{p-1}^{+}}\left[\sum_{l=0}^{k-1} P_{l}\{\mathrm{Sr}\}+\frac{1}{\xi_{k}} \sum_{l=0}^{k-2} P_{l}\{\mathrm{Sr}\}\right] \\
& =\cdots \\
& =\left[\frac{E\left[W_{0}, \mathrm{Sr}\right]}{\xi_{0}} \sum_{j=0}^{k} \prod_{l=0}^{j} \xi_{l}-\frac{1}{\lambda_{p-1}^{+}} \sum_{j=1}^{k}\left(\prod_{l=1}^{j} \xi_{l}\right)\left(\sum_{l=0}^{j-1} P_{l}\{\mathrm{Sr}\}\right) / / \prod_{j=1}^{k} \xi_{j} .\right.
\end{aligned}
$$

In order to determine $E\left[W_{0}, \mathrm{Sr}\right]$, we conjecture that

$$
\lim _{k \rightarrow \infty} E\left[W_{k}, \mathrm{Sr}\right]=0
$$

which yields

$$
E\left[W_{0}, \mathrm{Sr}\right]=\sum_{j=1}^{\infty}\left(\prod_{l=1}^{j} \xi_{l}\right)\left(\sum_{l=0}^{j-1} P_{l}\{\mathrm{Sr}\}\right) / m \mu \sum_{j=0}^{\infty} \prod_{l=0}^{j} \xi_{l}
$$


We then get

$$
\begin{aligned}
& E\left[W_{k}, \mathrm{Sr}\right]=\frac{\left(\sum_{j=0}^{k} \prod_{l=0}^{j} \xi_{l}\right)\left[\sum_{j=1}^{\infty}\left(\prod_{l=1}^{j} \xi_{l}\right)\left(\sum_{l=0}^{j-1} P_{l}\{\mathrm{Sr}\}\right)\right\rfloor}{m \mu\left(\sum_{j=0}^{\infty} \prod_{l=0}^{j} \xi_{l} \xi_{j}\right)\left(\sum_{j=1}^{k}\left(\sum_{l=0}^{j} \prod_{l=0}^{j} \xi_{l=0}^{j} \xi_{l} P_{l}\{\mathrm{Sr}\}\right)\right.} \\
& k \geq 1 \text {. }
\end{aligned}
$$

For customers of the highest priority class $p=1$, we have

$$
\begin{aligned}
& E\left[W_{0}, \mathrm{Sr}\right]=b_{0} P_{0}\{\mathrm{Sr}\} \\
& E\left[W_{k}, \mathrm{Sr}\right]=b_{k} P_{k}\{\mathrm{Sr}\}+\alpha_{k} E\left[W_{k-1}, \mathrm{Sr}\right] \quad k \geq 1,
\end{aligned}
$$

which is satisfied by

$$
E\left[W_{k}, \mathrm{Sr}\right]=\frac{\sum_{j=0}^{k} P_{j}\{\mathrm{Sr}\}}{m \mu+(k+1) \theta} \quad k \geq 0 .
$$

Finally, for a customer of class $p$, the joint probability that he is served and his mean waiting time and the conditional mean waiting time are given by

$$
E\left[W^{(p)}, \mathrm{Sr}\right]=\sum_{k=0}^{\infty} Q_{k} E\left[W_{k}, \mathrm{Sr}\right] \quad ; \quad E\left[W^{(p)} \mid \mathrm{Sr}\right]=\frac{E\left[W^{(p)}, \mathrm{Sr}\right]}{P\left\{\mathrm{Sr}^{(p)}\right\}}
$$

where $E\left[W_{k}, \mathrm{Sr}\right]$ in Eq. (25) is used for class $2 \leq p \leq P$ and $E\left[W_{k}, \mathrm{Sr}\right]$ in Eq. (26) is used for class $p=1$. Also, $P\left\{\mathrm{Sr}^{(p)}\right\}$ is given in Eq. (18).

Figure 7 plots $E\left[W^{(p)} \mid \mathrm{Sr}\right]$ for class $p=1$ through 4 against the arrival rate $\lambda$ for each class in the numerical example defined in Section 1. It is also tabulated in Table 2.

\subsection{Mean time to abandonment}

We denote by $E\left[W_{k}, \mathrm{Ab}\right]$ the joint probability that a customer of class $p$ who has $k$ other customers of classes $1,2, \ldots, p$ before him in the waiting room abandons waiting and the mean time to the abandonment, which is given by

$$
E\left[W_{k}, \mathrm{Ab}\right]=-W_{k}^{*(1)}(0, \mathrm{Ab}) \quad k \geq 0 .
$$




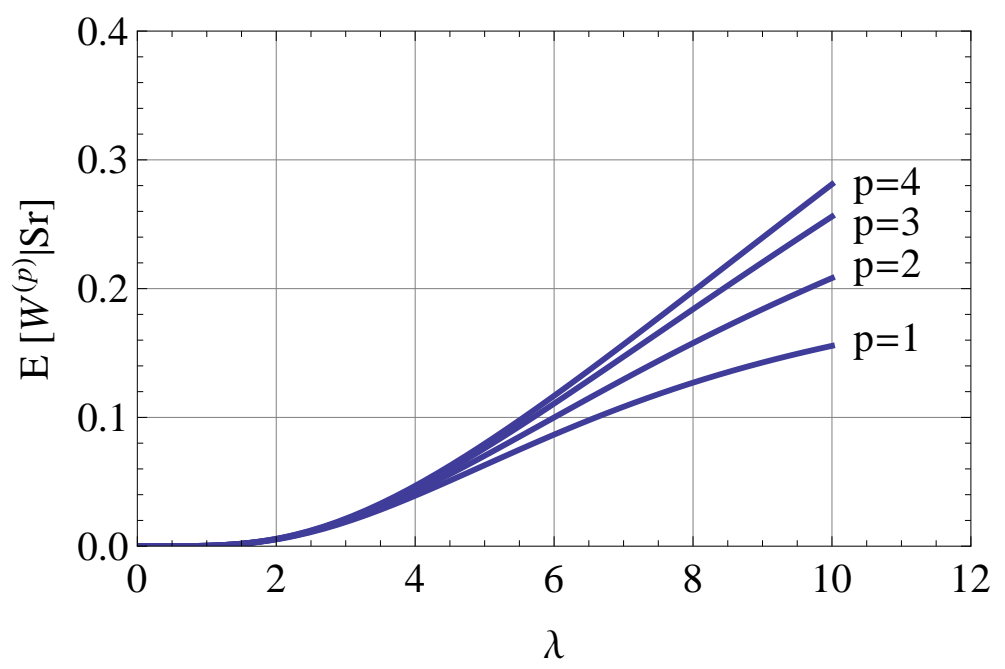

Figure 7: Mean waiting time for served customers of class $p$.

For customers of class $p(\geq 2)$, from Eq. (8) we can derive the following set of equations for $\left\{E\left[W_{k}, \mathrm{Ab}\right] ; k \geq 0\right\}$ :

$$
\begin{aligned}
E\left[W_{0}, \mathrm{Ab}\right] & =b_{0} P_{0}\{\mathrm{Ab}\}+\left(1-\alpha_{0}-\beta_{0}\right) E\left[W_{1}, \mathrm{Ab}\right], \\
E\left[W_{k}, \mathrm{Ab}\right] & =b_{k} P_{k}\{\mathrm{Ab}\}+\alpha_{k} E\left[W_{k-1}, \mathrm{Ab}\right] \\
& +\left(1-\alpha_{k}-\beta_{k}\right) E\left[W_{k+1}, \mathrm{Ab}\right] \quad k \geq 1 .
\end{aligned}
$$

We notice that these equations have the same coefficients as the equations for $\left\{E\left[W_{k}, \mathrm{Sr}\right] ; k \geq 0\right\}$ while $P_{k}\{\mathrm{Ab}\}$ appears in place of $P_{k}\{\mathrm{Sr}\}$ in the nonhomogeneous terms. We then get

$$
E\left[W_{0}, \mathrm{Ab}\right]=\sum_{j=1}^{\infty}\left(\prod_{l=1}^{j} \xi_{l}\right)\left(\sum_{l=0}^{j-1} P_{l}\{\mathrm{Ab}\}\right) / m \mu \sum_{j=0}^{\infty} \prod_{l=0}^{j} \xi_{l}
$$


Table 2: Mean waiting time of a served customer $E\left[W^{(p)} \mid \mathrm{Sr}\right]$.

\begin{tabular}{rcccc}
\hline$\lambda$ & $E\left[W^{(1)} \mid \mathrm{Sr}\right]$ & $E\left[W^{(2)} \mid \mathrm{Sr}\right]$ & $E\left[W^{(3)} \mid \mathrm{Sr}\right]$ & $E\left[W^{(4)} \mid \mathrm{Sr}\right]$ \\
\hline 0 & 0.0000 & 0.0000 & 0.0000 & 0.0000 \\
5 & 0.0629 & 0.0702 & 0.0760 & 0.0795 \\
10 & 0.1556 & 0.2082 & 0.2560 & 0.2810 \\
15 & 0.1957 & 0.3075 & 0.4163 & 0.4724 \\
20 & 0.2225 & 0.3947 & 0.5532 & 0.6269 \\
25 & 0.2491 & 0.4842 & 0.6737 & 0.7492 \\
30 & 0.2776 & 0.5772 & 0.7798 & 0.8493 \\
35 & 0.3081 & 0.6708 & 0.8729 & 0.9345 \\
40 & 0.3402 & 0.7621 & 0.9548 & 1.0089 \\
45 & 0.3735 & 0.8486 & 1.0273 & 1.0750 \\
50 & 0.4076 & 0.9291 & 1.0918 & 1.1343 \\
\hline
\end{tabular}

and

$$
E\left[W_{k}, \mathrm{Ab}\right]=\frac{\left(\sum_{j=0}^{k} \prod_{l=0}^{j} \xi_{l}\right]\left[\sum_{j=1}^{\infty}\left(\prod_{l=1}^{j} \xi_{l}\right)\left(\sum_{l=0}^{j-1} P_{l}\{\mathrm{Ab}\}\right)\right\rfloor}{\left.m \mu\left(\prod_{j=0}^{\infty} \prod_{l=0}^{j} \xi_{j}\right)\left(\sum_{j=0}^{k} \prod_{l=0}^{j} \xi_{l} \xi_{l=1}^{j} \xi_{l=1}^{j-1} P_{l}\{\mathrm{Ab}\}\right)\right\rfloor}
$$

For customers of the highest priority class $p=1$, we have

$$
\begin{aligned}
& E\left[W_{0}, \mathrm{Ab}\right]=b_{0} P_{0}\{\mathrm{Ab}\} \\
& E\left[W_{k}, \mathrm{Ab}\right]=b_{k} P_{k}\{\mathrm{Ab}\}+\alpha_{k} E\left[W_{k-1}, \mathrm{Ab}\right] \quad k \geq 1,
\end{aligned}
$$

which is satisfied by

$$
E\left[W_{k}, \mathrm{Ab}\right]=\frac{\sum_{j=0}^{k} P_{j}\{\mathrm{Ab}\}}{m \mu+(k+1) \theta} \quad k \geq 0 .
$$

Finally, for a customer of class $p$, the joint probability that he abandons waiting and the mean time to his abandonment and the conditional mean time 
to abandonment are given by

$$
E\left[W^{(p)}, \mathrm{Ab}\right]=\sum_{k=0}^{\infty} Q_{k} E\left[W_{k}, \mathrm{Ab}\right] \quad ; \quad E\left[W^{(p)} \mid \mathrm{Ab}\right]=\frac{E\left[W^{(p)}, \mathrm{Ab}\right]}{P\left\{\mathrm{Ab}^{(p)}\right\}} .
$$

where $E\left[W_{k}, \mathrm{Ab}\right]$ in Eq. (28) is used for class $2 \leq p \leq P$ and $E\left[W_{k}, \mathrm{Ab}\right]$ in Eq. (29) is used for class $p=1$. Also, $P\left\{\mathrm{Ab}^{(p)}\right\}$ is given in Eq. (22).

Figure 8 plots $E\left[W^{(p)} \mid \mathrm{Ab}\right]$ for class $p=1$ through 4 against the arrival rate $\lambda$ for each class in the numerical example defined in Section 1. It is also tabulated in Table 3.

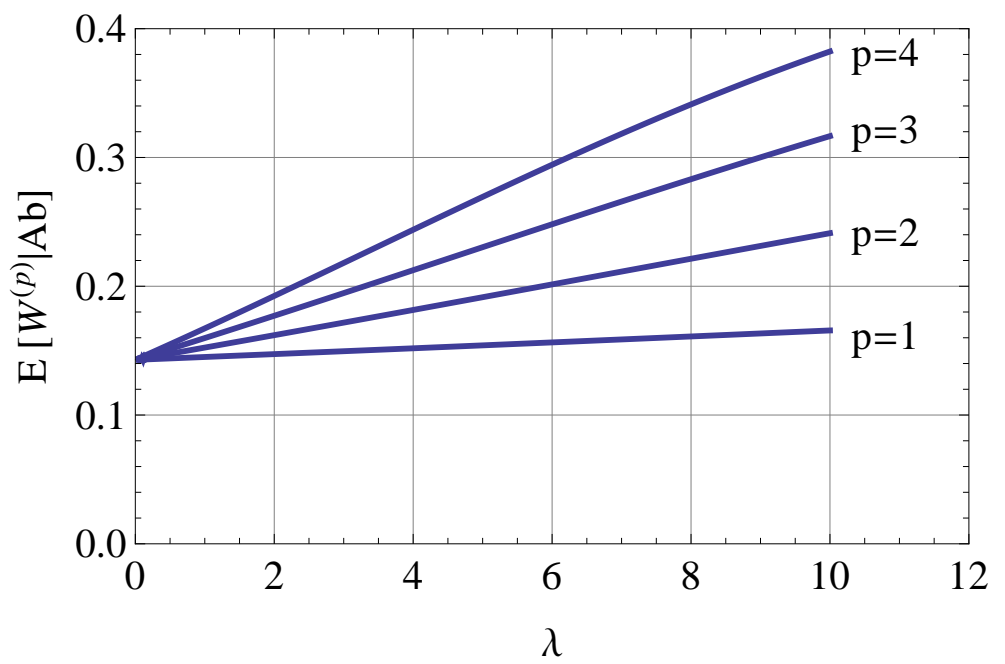

Figure 8: Mean time to abandonment for customers of class $p$.

\subsection{Unconditional mean waiting time}

We denote by $E\left[W_{k}\right]$ the mean time that a customer of class $p$ who has $k$ other customers of classes $1,2, \ldots, p$ before him spends in the waiting room until his service is started or he abandons waiting, whichever occurs first. This is given by

$$
E\left[W_{k}\right]=E\left[W_{k}, \mathrm{Sr}\right]+E\left[W_{k}, \mathrm{Ab}\right] \quad k \geq 0 .
$$

For customers of class $p(\geq 2)$, we have

$$
E\left[W_{0}\right]=\sum_{j=1}^{\infty} j \prod_{l=1}^{j} \xi_{l} / m \mu \sum_{j=0}^{\infty} \prod_{l=0}^{j} \xi_{l}
$$


Table 3: Mean time to abandonment $E\left[W^{(p)} \mid \mathrm{Ab}\right]$.

\begin{tabular}{rcccc}
\hline$\lambda$ & $E\left[W^{(1)} \mid \mathrm{Ab}\right]$ & $E\left[W^{(2)} \mid \mathrm{Ab}\right]$ & $E\left[W^{(3)} \mid \mathrm{Ab}\right]$ & $E\left[W^{(4)} \mid \mathrm{Ab}\right]$ \\
\hline 0 & 0.1429 & 0.1429 & 0.1429 & 0.1429 \\
5 & 0.1541 & 0.1915 & 0.2303 & 0.2694 \\
10 & 0.1657 & 0.2412 & 0.3166 & 0.3823 \\
15 & 0.1775 & 0.2894 & 0.3888 & 0.4534 \\
20 & 0.1893 & 0.3339 & 0.4400 & 0.4853 \\
25 & 0.2009 & 0.3729 & 0.4709 & 0.4961 \\
30 & 0.2124 & 0.4056 & 0.4872 & 0.4991 \\
35 & 0.2234 & 0.4319 & 0.4947 & 0.4998 \\
40 & 0.2341 & 0.4521 & 0.4980 & 0.5000 \\
45 & 0.2442 & 0.4672 & 0.4992 & 0.5000 \\
50 & 0.2537 & 0.4780 & 0.4997 & 0.5000 \\
\hline
\end{tabular}

and

$$
E\left[W_{k}\right]=\frac{\left(\sum_{j=0}^{k} \prod_{l=0}^{j} \xi_{l}\right)\left[\sum_{j=1}^{\infty} j\left(\prod_{l=1}^{j} \xi_{l}\right)\right]-\left(\sum_{j=0}^{\infty} \prod_{l=0}^{j} \xi_{l}\right)\left[\sum_{j=1}^{k} j\left(\prod_{l=1}^{j} \xi_{l}\right)\right]}{m \mu\left(\prod_{j=0}^{k} \xi_{j}\right)\left(\sum_{j=0}^{\infty} \prod_{l=0}^{j} \xi_{l}\right) \quad k \geq 1 . \quad(30)}
$$

The mean time that a customer of class $p$ spends in the waiting room is given by

$$
E\left[W^{(p)}\right]=\sum_{k=0}^{\infty} Q_{k} E\left[W_{k}\right] \quad 1 \leq p \leq P .
$$

However, by letting $s \rightarrow 0$ in Eq. (10), we have

$$
P_{k}\{\mathrm{Ab}\}=\theta E\left[W_{k}\right] \quad k \geq 0 .
$$

Substituting Eq. (32) into Eq. (31), we get the relation

$$
E\left[W^{(p)}\right]=\frac{1}{\theta} P\left\{\mathrm{Ab}^{(p)}\right\} \quad 1 \leq p \leq P,
$$

which also results from Eq. (13) or Eq. (14). From Eqs. (23) and (33), we confirm the Little's theorem for customers of class $p$ :

$$
E\left[L^{(p)}\right]=\lambda_{p} E\left[W^{(p)}\right] \quad 1 \leq p \leq P .
$$


We also note the relation

$$
E\left[W^{(p)}, \mathrm{Sr}\right]+E\left[W^{(p)}, \mathrm{Ab}\right]=E\left[W^{(p)}\right] \quad 1 \leq p \leq P,
$$

which can be used to check the accuracy of numerical computation.

Figure 9 plots $E\left[W^{(p)}\right]$ for class $p=1$ through 4 against the arrival rate $\lambda$ for each class in the numerical example defined in Section 1. It is also tabulated in Table 4.

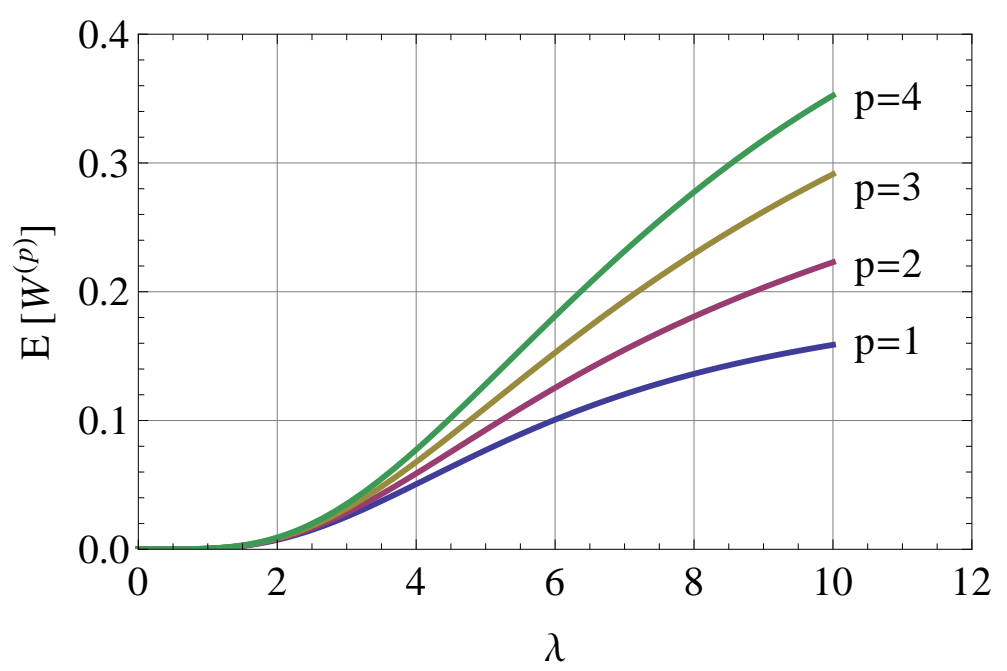

Figure 9: Unconditional mean waiting time for customers of class $p$.

\section{Second moment of the waiting time}

We can also study the second moment of the time that an arbitrary customer spends in the waiting room in the same way as for the mean waiting time.

\subsection{Second moment of the waiting time of served customers}

We denote by $E\left[W_{k}^{2}, \mathrm{Sr}\right]$ the joint probability that a customer of class $p$ who has $k$ other customers of classes $1,2, \ldots, p$ before him in the waiting room is eventually served and the second moment of his waiting time, which is given by

$$
E\left[W_{k}^{2}, \mathrm{Sr}\right]=W_{k}^{*(2)}(0, \mathrm{Sr}) \quad k \geq 0 .
$$


Table 4: Unconditional mean waiting time $E\left[W^{(p)}\right]$.

\begin{tabular}{rcccc}
\hline$\lambda$ & $E\left[W^{(1)}\right]$ & $E\left[W^{(2)}\right]$ & $E\left[W^{(3)}\right]$ & $E\left[W^{(4)}\right]$ \\
\hline 0 & 0.0000 & 0.0000 & 0.0000 & 0.0000 \\
5 & 0.0770 & 0.0927 & 0.1100 & 0.1282 \\
10 & 0.1588 & 0.2229 & 0.2913 & 0.3524 \\
15 & 0.1888 & 0.2968 & 0.3946 & 0.4551 \\
20 & 0.2087 & 0.3519 & 0.4511 & 0.4886 \\
25 & 0.2272 & 0.3960 & 0.4793 & 0.4974 \\
30 & 0.2456 & 0.4297 & 0.4919 & 0.4995 \\
35 & 0.2635 & 0.4539 & 0.4970 & 0.4999 \\
40 & 0.2806 & 0.4704 & 0.4989 & 0.5000 \\
45 & 0.2968 & 0.4814 & 0.4996 & 0.5000 \\
50 & 0.3117 & 0.4884 & 0.4999 & 0.5000 \\
60 & 0.3377 & 0.4957 & 0.5000 & 0.5000 \\
70 & 0.3587 & 0.4985 & 0.5000 & 0.5000 \\
80 & 0.3755 & 0.4995 & 0.5000 & 0.5000 \\
90 & 0.3891 & 0.4998 & 0.5000 & 0.5000 \\
100 & 0.4001 & 0.4999 & 0.5000 & 0.5000 \\
\hline
\end{tabular}

For customers of class $p(\geq 2)$, from Eq. (7) we can derive the following set of equations for $\left\{E\left[W_{k}^{2}, \mathrm{Sr}\right] ; k \geq 0\right\}$ :

$$
\begin{aligned}
E\left[W_{0}^{2}, \mathrm{Sr}\right] & =2 b_{0} E\left[W_{0}, \mathrm{Sr}\right]+\left(1-\alpha_{0}-\beta_{0}\right) E\left[W_{1}^{2}, \mathrm{Sr}\right] \\
E\left[W_{k}^{2}, \mathrm{Sr}\right] & =2 b_{k} E\left[W_{k}, \mathrm{Sr}\right]+\alpha_{k} E\left[W_{k-1}^{2}, \mathrm{Sr}\right] \\
& +\left(1-\alpha_{k}-\beta_{k}\right) E\left[W_{k+1}^{2}, \mathrm{Sr}\right] \quad k \geq 1,
\end{aligned}
$$

where $E\left[W_{k}, \mathrm{Sr}\right]$ is given in Eq. (25). These equations can be written as

$$
\begin{aligned}
E\left[W_{0}^{2}, \mathrm{Sr}\right]-\xi_{1} E\left[W_{1}^{2}, \mathrm{Sr}\right] & =-\eta_{0} \xi_{0} E\left[W_{0}^{2}, \mathrm{Sr}\right]+\frac{2 E\left[W_{0}, \mathrm{Sr}\right]}{m \mu+\theta} \\
E\left[W_{k}^{2}, \mathrm{Sr}\right]-\xi_{k+1} E\left[W_{k+1}^{2}, \mathrm{Sr}\right] & =\eta_{k}\left(E\left[W_{k-1}^{2}, \mathrm{Sr}\right]-\xi_{k} E\left[W_{k}^{2}, \mathrm{Sr}\right]\right) \\
& +\frac{2 E\left[W_{k}, \mathrm{Sr}\right]}{m \mu+(k+1) \theta} \quad k \geq 1 .
\end{aligned}
$$

The structure of these equations is the same as that for the equations for 
$\left\{E\left[W_{k}, \mathrm{Sr}\right] ; k \geq 0\right\}$ given in Section 5.1. Therefore, by analogy, we get

$$
\frac{E\left[W_{0}^{2}, \mathrm{Sr}\right]}{2}=\sum_{j=1}^{\infty}\left(\prod_{l=1}^{j} \xi_{l}\right)\left(\sum_{l=0}^{j-1} E\left[W_{l}, \mathrm{Sr}\right]\right) / m \mu \sum_{j=0}^{\infty} \prod_{l=0}^{j} \xi_{l}
$$

and

$$
\begin{aligned}
& \left(\sum_{j=0}^{k} \prod_{l=0}^{j} \xi_{l}\right)\left[\sum_{j=1}^{\infty}\left(\prod_{l=1}^{j} \xi_{l}\right)\left(\sum_{l=0}^{j-1} E\left[W_{l}, \mathrm{Sr}\right]\right)\right] \\
& \frac{E\left[W_{k}^{2}, \mathrm{Sr}\right]}{2}=\frac{-\left(\sum_{j=0}^{\infty} \prod_{l=0}^{j} \xi_{l}\right)\left[\sum_{j=1}^{k}\left(\prod_{l=1}^{j} \xi_{l}\right)\left(\sum_{l=0}^{j-1} E\left[W_{l}, \mathrm{Sr}\right]\right)\right]}{m \mu\left(\prod_{j=0}^{k} \xi_{j}\right)\left(\sum_{j=0}^{\infty} \prod_{l=0}^{j} \xi_{l}\right)} \\
& k \geq 1 .
\end{aligned}
$$

For customers of the highest priority class $p=1$, we have

$$
\begin{aligned}
& E\left[W_{0}^{2}, \mathrm{Sr}\right]=2 b_{0} E\left[W_{1}, \mathrm{Sr}\right], \\
& E\left[W_{k}^{2}, \mathrm{Sr}\right]=2 b_{k} E\left[W_{k}, \mathrm{Sr}\right]+\alpha_{k} E\left[W_{k-1}^{2}, \mathrm{Sr}\right] \quad k \geq 1,
\end{aligned}
$$

where $E\left[W_{k}, \mathrm{Sr}\right]$ is given in Eq. (26). These equations are satisfied by

$$
E\left[W_{k}^{2}, \mathrm{Sr}\right]=\frac{2 \sum_{j=0}^{k} E\left[W_{j}, \mathrm{Sr}\right]}{m \mu+(k+1) \theta} \quad k \geq 0 .
$$

\subsection{Second moment of the time to abandonment}

We denote by $E\left[W_{k}^{2}, \mathrm{Ab}\right]$ the joint probability that a customer of class $p$ who has $k$ other customers of classes $1,2, \ldots, p$ before him in the waiting room abandons waiting and the second moment of the time to his abandonment, which is given by

$$
E\left[W_{k}^{2}, \mathrm{Ab}\right]=W_{k}^{*(2)}(0, \mathrm{Ab}) \quad k \geq 0 .
$$

For customers of class $p(\geq 2)$, from Eq. (8) we can derive the following set of equations for $\left\{E\left[W_{k}^{2}, \mathrm{Ab}\right] ; k \geq 0\right\}$ :

$$
E\left[W_{0}^{2}, \mathrm{Ab}\right]=2 b_{0} E\left[W_{0}, \mathrm{Ab}\right]+\left(1-\alpha_{0}-\beta_{0}\right) E\left[W_{1}^{2}, \mathrm{Ab}\right],
$$




$$
\begin{aligned}
E\left[W_{k}^{2}, \mathrm{Ab}\right] & =2 b_{k} E\left[W_{k}, \mathrm{Ab}\right]+\alpha_{k} E\left[W_{k-1}^{2}, \mathrm{Ab}\right] \\
& +\left(1-\alpha_{k}-\beta_{k}\right) E\left[W_{k+1}^{2}, \mathrm{Ab}\right] \quad k \geq 1
\end{aligned}
$$

where $E\left[W_{k}, \mathrm{Ab}\right]$ is given in Eq. (28). Then we get

$$
\frac{E\left[W_{0}^{2}, \mathrm{Ab}\right]}{2}=\sum_{j=1}^{\infty}\left(\prod_{l=1}^{j} \xi_{l}\right)\left(\sum_{l=0}^{j-1} E\left[W_{l}, \mathrm{Ab}\right]\right) / m \mu \sum_{j=0}^{\infty} \prod_{l=0}^{j} \xi_{l}
$$

and

$$
\frac{\left(\sum_{j=0}^{k} \prod_{l=0}^{j} \xi_{l}\right)\left[\sum_{j=1}^{\infty}\left(\prod_{l=1}^{j} \xi_{l}\right)\left(\sum_{l=0}^{j-1} E\left[W_{l}, \mathrm{Ab}\right]\right)\right]}{m \mu\left(\prod_{j=0}^{\infty} \xi_{j}\right)\left(\sum_{j=0}^{\infty} \prod_{l=0}^{j} \xi_{l=0}^{j} \xi_{l}\right)\left[\sum_{j=1}^{k}\left(\prod_{l=1}^{j} \xi_{l}\right)\left(\sum_{l=0}^{j-1} E\left[W_{l}, \mathrm{Ab}\right]\right)\right]}
$$

For customers of the highest priority class $p=1$, we have

$$
\begin{aligned}
& E\left[W_{0}^{2}, \mathrm{Ab}\right]=2 b_{0} E\left[W_{1}, \mathrm{Ab}\right] \\
& E\left[W_{k}^{2}, \mathrm{Ab}\right]=2 b_{k} E\left[W_{k}, \mathrm{Ab}\right]+\alpha_{k} E\left[W_{k-1}^{2}, \mathrm{Ab}\right] \quad k \geq 1,
\end{aligned}
$$

where $E\left[W_{k}, \mathrm{Ab}\right]$ is given in Eq. (29). These equations are satisfied by

$$
E\left[W_{k}^{2}, \mathrm{Ab}\right]=\frac{2 \sum_{j=0}^{k} E\left[W_{j}, \mathrm{Ab}\right]}{m \mu+(k+1) \theta} \quad k \geq 0 .
$$

\subsection{Unconditional second moment of the waiting time}

We denote by $E\left[W_{k}^{2}\right]$ the second moment of the time that a customer of class $p$ who has $k$ other customers of classes $1,2, \ldots, p$ before him in the waiting room spends in the waiting room until his service is started or he abandons waiting, which is given by

$$
E\left[W_{k}^{2}\right]=E\left[W_{k}^{2}, \mathrm{Sr}\right]+E\left[W_{k}^{2}, \mathrm{Ab}\right] \quad k \geq 0 .
$$


For customers of class $p(\geq 2)$, we have

$$
E\left[W_{0}^{2}\right]=2 \sum_{j=1}^{\infty}\left(\prod_{l=1}^{j} \xi_{l}\right)\left(\sum_{l=0}^{j-1} E\left[W_{l}\right]\right) / m \mu \sum_{j=0}^{\infty} \prod_{l=0}^{j} \xi_{l}
$$

and

$$
\begin{aligned}
& \left(\sum_{j=0}^{k} \prod_{l=0}^{j} \xi_{l}\right)\left[\sum_{j=1}^{\infty}\left(\prod_{l=1}^{j} \xi_{l}\right)\left(\sum_{l=0}^{j-1} E\left[W_{l}\right]\right)\right] \\
& \frac{E\left[W_{k}^{2}\right]}{2}=\frac{-\left(\sum_{j=0}^{\infty} \prod_{l=0}^{j} \xi_{l}\left[\sum_{j=1}^{k}\left(\prod_{l=1}^{j} \xi_{l}\right)\left(\sum_{l=0}^{j-1} E\left[W_{l}\right]\right)\right\rfloor\right.}{m \mu\left(\prod_{j=0}^{k} \xi_{j}\right)\left(\sum_{j=0}^{\infty} \prod_{l=0}^{j} \xi_{l}\right)} \\
& k \geq 1,
\end{aligned}
$$

where $E\left[W_{l}\right]$ is given in Eq. (30).

The second moment of the time that a customer of class $p$ spends in the waiting room is given by

$$
E\left[\left\{W^{(p)}\right\}^{2}\right]=\sum_{k=0}^{\infty} Q_{k} E\left[W_{k}^{2}\right] \quad 1 \leq p \leq P .
$$

However, from Eq. (10), we have

$$
E\left[W_{k}, \mathrm{Ab}\right]=\frac{\theta}{2} E\left[W_{k}^{2}\right] \quad k \geq 0
$$

Substituting Eq. (42) into Eq. (41), we get the relation

$$
E\left[\left\{W^{(p)}\right\}^{2}\right]=\frac{2}{\theta} E\left[W^{(p)}, \mathrm{Ab}\right] \quad 1 \leq p \leq P,
$$

which also results from Eq. (13) or Eq. (14).

Figure 10 plots $E\left[\left\{W^{(p)}\right\}^{2}\right]$ for class $p=1$ through 4 against the arrival rate $\lambda$ for each class in the numerical example defined in Section 1. It is also tabulated in Table 5 . 


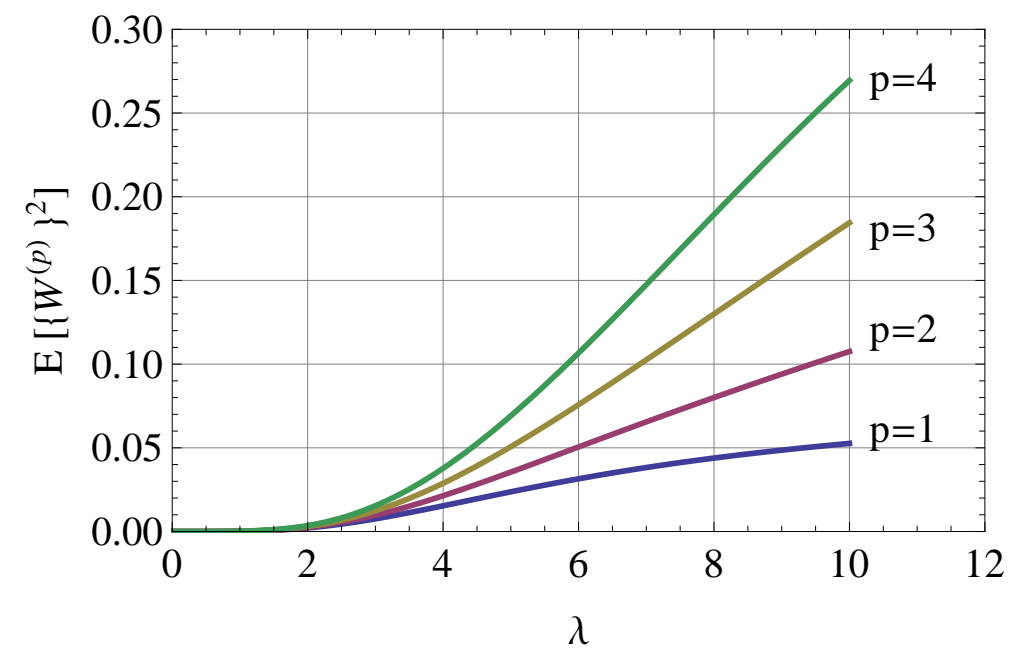

Figure 10: Second moment of the waiting time for customers of class $p$.

\section{Distribution of the waiting time for customers of the highest priority class}

It is straightforward to find the explicit expressions for $W_{k}^{*}(s, \mathrm{Sr})$ and $W_{k}^{*}(s, \mathrm{Ab})$ as the solution to Eqs. (11) and (12), respectively, for customers of the highest priority class $p=1$. The simplification results from the fact that their waiting times are not influenced by the arrivals of other customers of any classes who arrive after them. However, they are influenced by all other customers who are already in service when they arrive.

From Eq. (11), we get [13]

$$
W_{k}^{*}(s, \mathrm{Sr})=\frac{m \mu}{m \mu+(k+1) \theta} \prod_{j=0}^{k} B_{j}^{*}(s) \quad k \geq 0 .
$$

Then we obtain

$$
\begin{aligned}
W^{*}(s, \mathrm{Sr}) & =P\{W=0\}+\sum_{k=0}^{\infty} Q_{k} W_{k}^{*}(s, \mathrm{Sr}) \\
& =P\{W=0\}+Q_{0} \frac{m \mu}{\lambda_{1}} \sum_{k=1}^{\infty} \frac{\left(\lambda_{1}\right)^{k}}{\prod_{j=1}^{k}(s+m \mu+j \theta)},
\end{aligned}
$$


Table 5: Second moment of the waiting time $E\left[\left\{W^{(p)}\right\}^{2}\right]$.

\begin{tabular}{rcccc}
\hline$\lambda$ & $E\left[\left\{W^{(1)}\right\}^{2}\right]$ & $E\left[\left\{W^{(2)}\right\}^{2}\right]$ & $E\left[\left\{W^{(3)}\right\}^{2}\right]$ & $E\left[\left\{W^{(4)}\right\}^{2}\right]$ \\
\hline 0 & 0.0000 & 0.0000 & 0.0000 & 0.0000 \\
5 & 0.0237 & 0.0355 & 0.0507 & 0.0691 \\
10 & 0.0526 & 0.1075 & 0.1844 & 0.2695 \\
15 & 0.0670 & 0.1718 & 0.3068 & 0.4127 \\
20 & 0.0790 & 0.2350 & 0.3969 & 0.4742 \\
25 & 0.0913 & 0.2953 & 0.4515 & 0.4936 \\
30 & 0.1043 & 0.3486 & 0.4793 & 0.4986 \\
35 & 0.1178 & 0.3920 & 0.4918 & 0.4997 \\
40 & 0.1314 & 0.4254 & 0.4969 & 0.4999 \\
45 & 0.1449 & 0.4498 & 0.4989 & 0.5000 \\
50 & 0.1582 & 0.4670 & 0.4996 & 0.5000 \\
\hline
\end{tabular}

where $P\{W=0\}$ and $Q_{0}$ are given in Eqs. (3) and (4), respectively. This can be inverted to the joint probability of successful service and the probability density function (pdf) $f_{W}(t, \mathrm{Sr})$ for the waiting time such that

$$
W^{*}(s, \mathrm{Sr})=P\{W=0\}+\int_{0}^{\infty} e^{-s t} f_{W}(t, \mathrm{Sr}) d t,
$$

where

$$
f_{W}(t, \mathrm{Sr})=Q_{0} m \mu \exp \left[-(m \mu+\theta) t+\frac{\lambda_{1}}{\theta}\left(1-e^{-\theta t}\right)\right] \quad t>0 .
$$

This yields

$$
\begin{aligned}
P\{\mathrm{Sr}\} & =P\{W=0\}+\int_{0}^{\infty} f_{W}(t, \mathrm{Sr}) d t \\
E\left[W^{\ell}, \mathrm{Sr}\right] & =\int_{0}^{\infty} t^{\ell} f_{W}(t, \mathrm{Sr}) d t \quad \ell=1,2, \ldots
\end{aligned}
$$

It can be shown that Eq. (12) is satisfied by

$$
W_{k}^{*}(s, \mathrm{Ab})=\frac{\theta}{s+\theta}\left[1-W_{k}^{*}(s, \mathrm{Sr})\right] \quad k \geq 0,
$$

so that

$$
W^{*}(s, \mathrm{Ab})=\sum_{k=0}^{\infty} Q_{k} W_{k}^{*}(s, \mathrm{Ab})=\frac{\theta}{s+\theta}\left[1-W^{*}(s, \mathrm{Sr})\right]
$$


This is inverted to the joint probability of abandonment and the pdf for the time to abandonment given by

$$
f_{W}(t, \mathrm{Ab})=\theta e^{-\theta t}\left[P\{W>0\}-\int_{0}^{t} e^{\theta x} f_{W}(x, \mathrm{Sr}) d x\right] \quad t \geq 0
$$

so that

$$
W^{*}(s, \mathrm{Ab})=\int_{0}^{\infty} e^{-s t} f_{W}(t, \mathrm{Ab}) d t
$$

This yields

$$
\begin{aligned}
& P\{\mathrm{Ab}\}=\int_{0}^{\infty} f_{W}(t, \mathrm{Ab}) d t \quad ; \quad E\left[W^{\ell}, \mathrm{Ab}\right]=\int_{0}^{\infty} t^{\ell} f_{W}(t, \mathrm{Ab}) d t \\
& \ell=1,2, \ldots .
\end{aligned}
$$

Finally, the pdf for the unconditional waiting time is given by

$$
f_{W}(t)=f_{W}(t, \mathrm{Sr})+f_{W}(t, \mathrm{Ab}) \quad t>0,
$$

which corresponds to the Laplace transform

$$
W^{*}(s)=P\{W=0\}+\int_{0}^{\infty} e^{-s t} f_{W}(t) d t .
$$

We plot $f_{W}(t, \mathrm{Sr})$ and $f_{W}(t, \mathrm{Ab})$ for the case $\lambda_{1}=2$ in Figure 11.

\section{Concluding remarks}

In this paper, we have derived the explicit formulas for the probabilities of service and abandonment as well as the mean and second moment of the waiting time for every class. Actually it takes considerable amount of time to compute them from their direct expressions individually. For plotting, some of them can be computed more quickly by utilizing the relations among them as follows. Starting with $E\left[L^{(p)}\right]$ in Eq. (5), we can compute $P\left\{\mathrm{Ab}^{(p)}\right\}$ by Eq. (23), and then $P\left\{\mathrm{Sr}^{(p)}\right\}$ by Eq. (22). We can also obtain $E\left[W^{(p)}\right]$ by Eq. (34). The accuracy of computation can be checked against the calculation of direct expressions for selected parameter values as well as the confirmation of the relation in Eq. (19). Furthermore, once we obtain $E\left[W^{(p)}, \mathrm{Ab}\right]$, we can get $E\left[W^{(p)}, \mathrm{Sr}\right]$ by Eq. (35) and $E\left[\left\{W^{(p)}\right\}^{2}\right]$ by Eq. (43).

In the numerical examples considered above, as $\lambda$ increases, $P\left\{\mathrm{Ab}^{(p)}\right\}$, $E\left[W^{(p)}\right], E\left[W^{(p)} \mid \mathrm{Sr}\right], E\left[W^{(p)} \mid \mathrm{Ab}\right]$, and $E\left[\left\{W^{(p)}\right\}^{2}\right]$ increase monotonically 


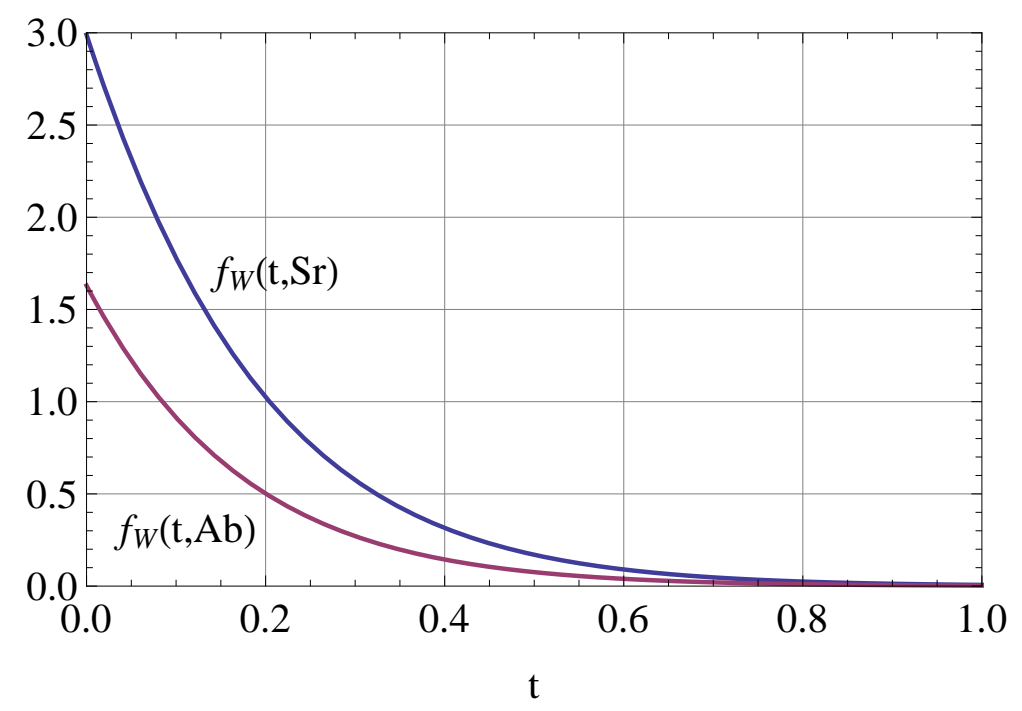

Figure 11: Probability density functions of the waiting time for customers of class 1 .

for each class $p$. We observe that they are larger for lower priority classes given the arrival rate $\lambda$ :

$$
\begin{aligned}
& P\left\{\mathrm{Ab}^{(1)}\right\}<P\left\{\mathrm{Ab}^{(2)}\right\}<P\left\{\mathrm{Ab}^{(3)}\right\}<P\left\{\mathrm{Ab}^{(4)}\right\}, \\
& E\left[W^{(1)}\right]<E\left[W^{(2)}\right]<E\left[W^{(3)}\right]<E\left[W^{(4)}\right], \\
& E\left[W^{(1)} \mid \mathrm{Sr}\right]<E\left[W^{(2)} \mid \mathrm{Sr}\right]<E\left[W^{(3)} \mid \mathrm{Sr}\right]<E\left[W^{(4)} \mid \mathrm{Sr}\right], \\
& E\left[W^{(1)} \mid \mathrm{Ab}\right]<E\left[W^{(2)} \mid \mathrm{Ab}\right]<E\left[W^{(3)} \mid \mathrm{Ab}\right]<E\left[W^{(4)} \mid \mathrm{Ab}\right], \\
& E\left[\left\{W^{(1)}\right\}^{2}\right]<E\left[\left\{W^{(2)}\right\}^{2}\right]<E\left[\left\{W^{(3)}\right\}^{2}\right]<E\left[\left\{W^{(4)}\right\}^{2}\right] .
\end{aligned}
$$

In general, we have the following limiting values for all classes. In the limit $\lambda \rightarrow 0$, we have

$$
\begin{aligned}
& \lim _{\lambda \rightarrow 0} P\left\{\mathrm{Ab}^{(p)}\right\}=\lim _{\lambda \rightarrow 0} E\left[W^{(p)}\right]=\lim _{\lambda \rightarrow 0} E\left[\left\{W^{(p)}\right\}^{2}\right]=0, \\
& \lim _{\lambda \rightarrow 0} E\left[W^{(p)} \mid \mathrm{Sr}\right]=0 ; \quad \lim _{\lambda \rightarrow 0} E\left[W^{(p)} \mid \mathrm{Ab}\right]=\frac{1}{m \mu+\theta} .
\end{aligned}
$$


In the limit $\lambda \rightarrow \infty$, we have

$$
\begin{aligned}
& \lim _{\lambda \rightarrow \infty} P\left\{\mathrm{Ab}^{(p)}\right\}=1 \quad ; \quad \lim _{\lambda \rightarrow \infty} E\left[W^{(p)}\right]=\frac{1}{\theta}, \\
& \lim _{\lambda \rightarrow \infty} E\left[W^{(p)} \mid \mathrm{Ab}\right]=\frac{1}{\theta} \quad ; \quad \lim _{\lambda \rightarrow \infty} E\left[\left\{W^{(p)}\right\}^{2}\right]=\frac{2}{\theta^{2}} .
\end{aligned}
$$

However, at this moment we do not know about the limit

$$
\lim _{\lambda \rightarrow \infty} E\left[W^{(p)} \mid \mathrm{Sr}\right] .
$$

We have assumed the first-come, first-served (FCFS) discipline within the same class in this paper. We can treat the case of last-come, first-served (LCFS) discipline within the same class as well in a similar fashion. It turns out that the probabilities of service and abandonment, $P\left\{\mathrm{Sr}^{(p)}\right\}$ and $P\left\{\mathrm{Ab}^{(p)}\right\}$, and the unconditional mean waiting time $E\left[W^{(p)}\right]$ are the same but that the conditional mean waiting times, $E\left[W^{(p)} \mid \mathrm{Sr}\right]$ and $E\left[W^{(p)} \mid \mathrm{Ab}\right]$, and the unconditional second moment of the waiting time $E\left[\left\{W^{(p)}\right\}^{2}\right]$ are different for the FCFS and LCFS systems. A detailed analysis of the waiting time in the $\mathrm{M} / \mathrm{M} / m$ LCFS nonpreemptive priority queue with impatient customers will be presented elsewhere.

\section{References}

[1] A. Brandt and M. Brandt, On a two-queue priority system with impatience and its application to a call center, Methodology and Computing in Applied Probability, 1, No.2 (1999), 191-210. DOI: http://dx.doi.org/10.1023/A:1010009304213

[2] A. Cobham, Priority assignment in waiting line problems, Operations Research, 2, No.1 (1954), 70-76. DOI: http://dx.doi.org/10.1287/opre.2.1.70

[3] R. H. Davis, Waiting-time distribution of a multi-server, priority queuing system, Operations Research, 14, No.1 (1966), 133-136. DOI: http://dx.doi.org/10.1287/opre.14.1.133

[4] B. V. Gnedenko and I. N. Kovalenko, Introduction to Queueing Theory, Second edition revised and supplemented. Translated by Samuel Kotz, Birkhäuser Boston, 1989. 
[5] F. Iravani and B. Balcıoglu, On priority queues with impatient customers, Queueing Systems, 58, No.4 (2008), 239-260. DOI: http://dx.doi.org/10.1007/s11134-008-9069-6

[6] O. Kella and U. Yechiali, Waiting times in the non-preemptive priority M/M/c queue. Stochastic Models, 1, No.2 (1985), 257-262. DOI: http://dx.doi.org/10.1080/15326348508807014

[7] V. G. Kulkarni, Modeling and Analysis of Stochastic Systems, Chapman \& Hall, 1995.

[8] A. Mandelbaum and S. Zeltyn, Service engineering in action: The Palm/Erlang-A queue, with applications to call centers. In D. Spath and K.-P. Fähnrich (eds.): Advances in Services Innovations, Springer, Berlin, (2007), 17-45.

[9] A. Myskja and O. Espvik (editors), Tore Olaus Engset, 1865-1943, The Man Behind the Formula, Tapir Academic Press, 2002.

[10] C. Palm, Etude des délais d'attente, Ericsson Technics, 5, No.2 (1937), $39-56$, cited in [12].

[11] C. Palm, Research on telephone traffic carried by full availability groups. Tele, 1 (1957), 1-107 (English translation of results first published in 1946 in Swedish in the same journal, which was then entitled Tekniska Meddelanden från Kungliga Telegrafstyrelsen.), cited in [8] and [12].

[12] J. Riordan, Stochastic Service Systems, John Wiley \& Sons, 1962.

[13] H. Takagi, Waiting time in the $\mathrm{M} / \mathrm{M} / m /(m+c)$ queue with impatient customers. International Journal of Pure and Applied Mathematics, 90, No.4 (2014), 519-559. DOI: http://dx.doi.org/10.12732/ijpam.v90i4.13

[14] H. M. Taylor and S. Karlin, An Introduction to Stochastic Modeling, Third edition, Academic Press, 1998.

[15] W. Whitt, Engineering solution of a basic call-center model, Management Science, $\mathbf{5 1}, \quad$ No.2 (2005), 221-235. DOI: http://dx.doi.org/10.1287/mnsc.1040.0302. 